To appear in the Journal of Mathematics and the Arts

Vol. 00, No. 00, Month 20XX, 1-26

\title{
Exploring symmetry in rosettes of Truchet tiles
}

\author{
Andreia Hall ${ }^{a *}$, Paulo Almeida ${ }^{a}$ \\ and Ricardo Teixeira ${ }^{b}$ \\ ${ }^{a}$ CIDMA - Center for Research and Development in Mathematics and Applications, Department of \\ Mathematics, University of Aveiro, Aveiro, Portugal; \\ ${ }^{b}$ NICA - Interdisciplinary Center for Childhood and Adolescence, Faculty of Sciences and Technology, \\ University of the Azores, Ponta Delgada, Portugal
}

(v4.0 released April 2013)

\begin{abstract}
In 1704 the French priest Sébastien Truchet published a paper where he explored and counted patterns made up from a square divided by a diagonal line into two colored parts,

a Truchet tile. A few years later, Father Dominique Doüat continued Truchet's work and book in 1722 containing many more patterns and further counts of configurations.

In this paper, we extend the work introduced by Truchet and Doüat by considering all possible rosettes made up of an $m \times n$ array of square or non-square Truchet tiles, $\boldsymbol{\Lambda}$ or $\square$. We then classify the rosettes according to their symmetry group and count all the distinct rosettes in each group, for all possible sizes. The results are summarized in a separate section where we further analyze the asymptotic behavior of the counts for square arrays. Finally, some applications are shown using two types of square flexagons.
\end{abstract}

Keywords: Truchet tiles; rosette symmetry groups; recreational mathematics.

AMS Subject Classification: F1.1; F4.3.

\section{Introduction}

The intuitive concept of symmetry has accompanied us since childhood, from the time we became aware of the world around us. It is possible to find countless examples of symmetry in nature (a butterfly's wings, the petals of a flower, or a starfish), in architecture, in decorative art, and in many objects that we encounter on a daily basis [11].

Puzzled with the idea of symmetry, man has tried to comprehend and create order through the ages. In all cultures of the world, including those dating back to prehistoric times, humans have developed an intuitive understanding of symmetry, interpreting it as a harmony of proportions [13. Temples, tombs and other structures were often designed with particular attention to symmetry, harmony and balance.

Generically, the concept of symmetry expresses harmony and balance. But it may also be studied rigorously from a mathematical point of view. In this case, the mathematician's work consists of finding, studying and classifying all kinds of patterns. This strenuous task helps us to better understand interesting cultural aspects of our existence as a species.

To simplify the classification of patterns, examples of decorative and ornamental art belonging to the 3-dimensional world will be considered as if they were sets of points on a 2-dimensional plane. A symmetry of a figure is an isometry of the plane that maps the figure back onto itself.

*Corresponding author. Email: andreia.hall@ua.pt 
For example, if you consider a square and rotate the plane 90 degrees around its center, you still get a square in exactly the same position as you started. We say the square has a rotational symmetry of 90 degrees.

There are four types of symmetry: reflection symmetry or mirror symmetry (associated to a line, called the axis of symmetry); rotational symmetry (associated to a point, called the rotation center, and to a given angle); translational symmetry (associated to a vector, with a given direction and magnitude); and glide reflection symmetry (resulting from the composition of a reflection in a line and a translation with the direction of that line).

The classification of figures based on its symmetries has significant practical implications and is an excellent opportunity to show how mathematics, as a science of patterns, plays a fundamental role in the organization of information around us. The set of all symmetries of a figure forms a group under composition, the symmetry group of that figure. In the plane there are only three categories of discrete symmetry groups [8]: rosette groups (they have a finite number of symmetries which can only be rotations or reflections); frieze groups (they have translational symmetries in only one direction; one can identify a unit cell which is replicated at a constant distance along a straight line); and wallpaper groups (they have translational symmetries in two directions, which lead to the paving of the plane).

The symmetry group of a rosette can be a cyclic group $C_{n}$ (figures with $n$ rotational symmetries) or a dihedral group $D_{n}$ (figures with $n$ rotational symmetries and $n$ mirror symmetries). The rotational symmetries all have the same center and are associated with rotation angles of $360 / n$ degrees and multiples thereof. The axes of symmetry, if any, all pass through the center of rotation (Figure 1). Note that except for the rosettes with symmetry group $C_{1}$, which only have the identity transformation and therefore do not possess any type of symmetry, all other rosettes exhibit some type of symmetry (rotational and/or reflection symmetry). For more

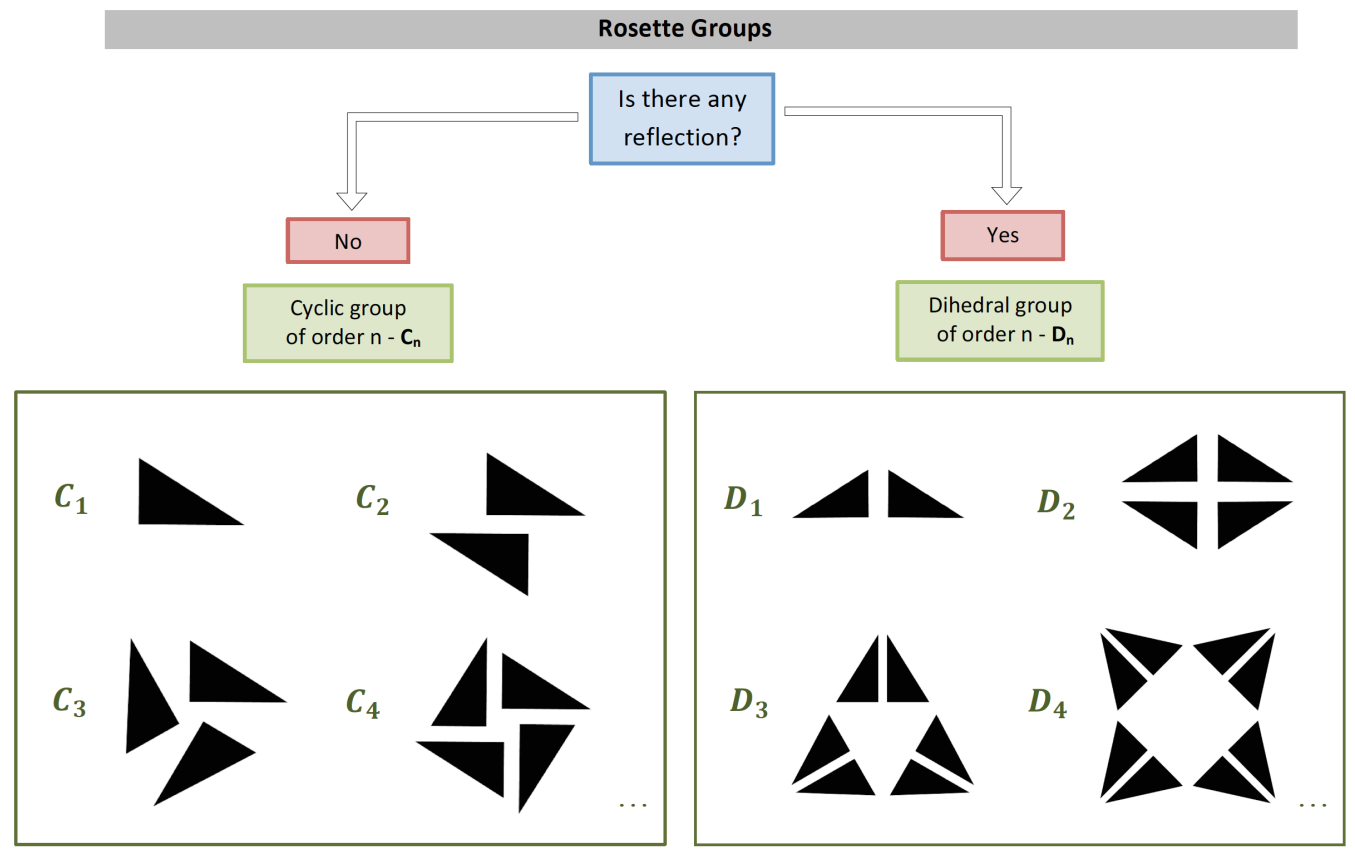

Figure 1. Flowchart for the classification of a rosette.

details on symmetries, see [4, 8, 12, 13].

With only one motif it is possible to generate a great diversity of patterns with different symmetry groups. For instance, in Figure1 we use only one motif: a right triangle. This principle of modularity is very old, dating back to the Paleolithic (23000 B.C.) and Neolithic (5000 B.C.) ornamental art, passing through the Celtic, ancient Greek and Roman ornaments among many others [6] and being still widely used in more recent contexts, such as the OpArt movement of 
the twentieth century and computer generated images of the present day. It is interesting to note that while in past centuries symmetry was an important feature in modular ornaments, more recently modularity is often used through random generators creating very different, though not less interesting, patterns. In Portugal, modularity is common, for instance, in buildings covered with traditional ceramic tiles. The azulejo, Portuguese word for tile, is nowadays regarded as one of the most original creations of the Portuguese culture, for the immense heritage existing all over the country and what were once the overseas territories, in Brazil, Africa and India.

A simple and common module is the Truchet tile - a square, or more generally a rectangle, divided by a diagonal line into two colored parts, $\boldsymbol{\Lambda}$ or $\boldsymbol{~ T r u c h e t ~ t i l e s ~ c a n ~ b e ~ f o u n d ~ i n ~ s e v e r a l ~}$ ceramic facades. In Porto more than 40 buildings are covered with square Truchet tiles. Figure 2 shows one such example of a building in Porto and another example from Lagoa, in the Azores Islands.
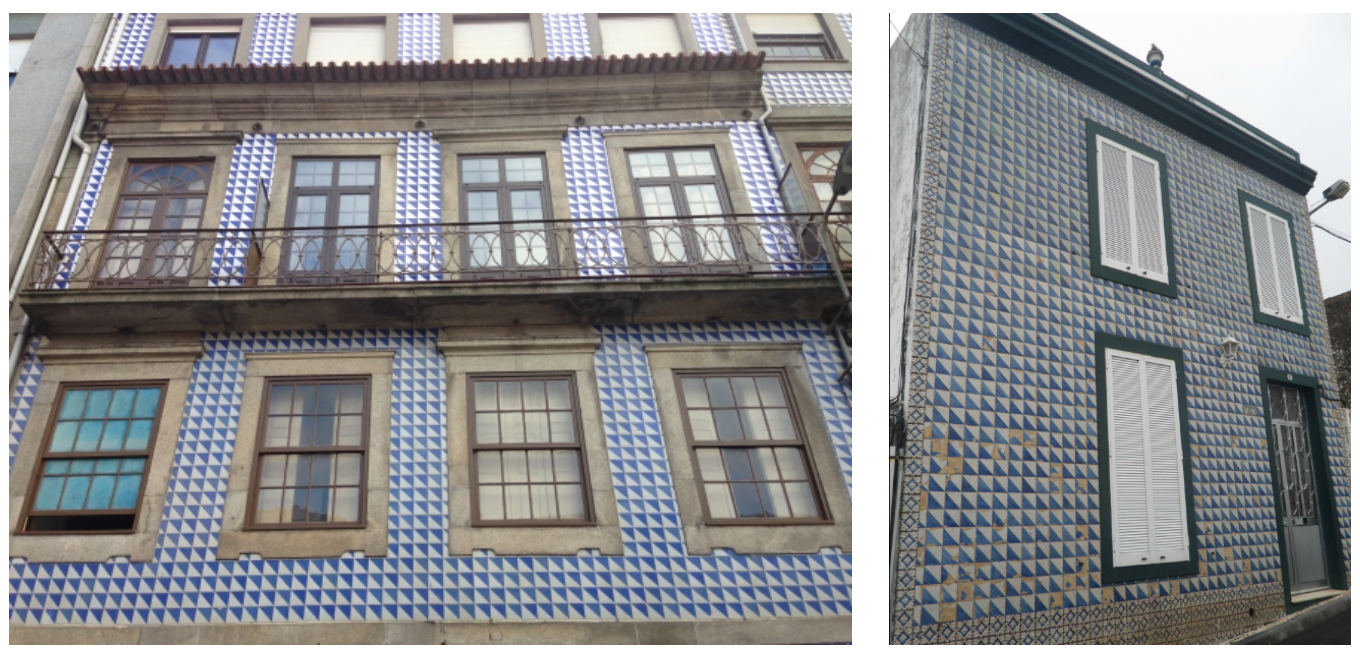

Figure 2. Buildings coverred with square Truchet tiles in Porto and Lagoa, Azores.

In different contexts throughout our history, one can find many examples of patterns (rosettes, friezes and wallpapers) that can be made with Truchet tiles. Some interesting examples of friezes come from Neolithic ornamental art (Figure 3). For these examples and more, see [5].

(a)

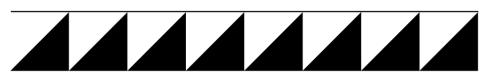

(c)

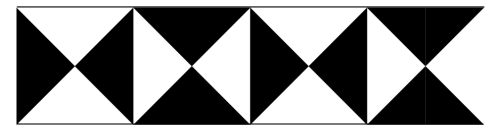

(b)

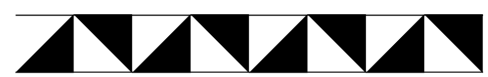

(d)

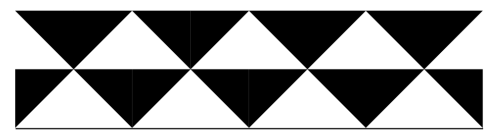

Figure 3. Examples from Neolithic ornamental art: (a) Near East, about 5000 B.C.; (b) Near East, about 5000 B.C.; (c) Tell el Hallaf, about 4900-4500 B.C.; (d) Hadlar, about 5500-5200 B.C.

Figure 4 illustrates examples of friezes and wallpapers from different cultures [11, 13].

Truchet tiles were named after Sébastien Truchet (1657-1729), a French catholic priest. He was active in areas such as mathematics, hydraulics and typography. Truchet studied decorative patterns made by square Truchet tiles. He observed that many different patterns could be formed by placing these tiles in different orientations with respect to each other. Some of these configurations were published in his article Memoir sur les Combinasions (Memoires de l'Académie Royale des Sciences, 1704), and are reprinted in [10. Figure 5 shows three of those 
(a)

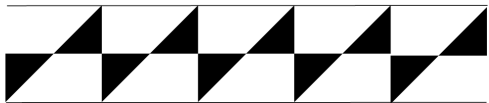

(b)

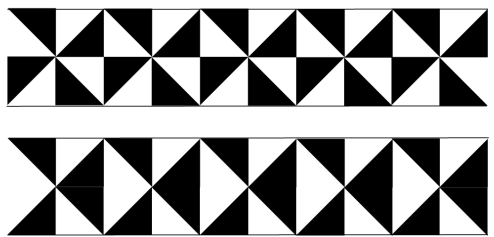

(c)

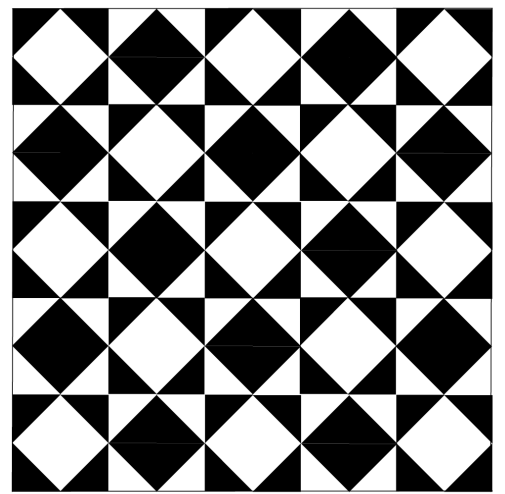

Figure 4. (a) Navarro Indian Art; (b) Tapa Cloth from Fiji; (c) Rafter lashings made for a Tongan church.
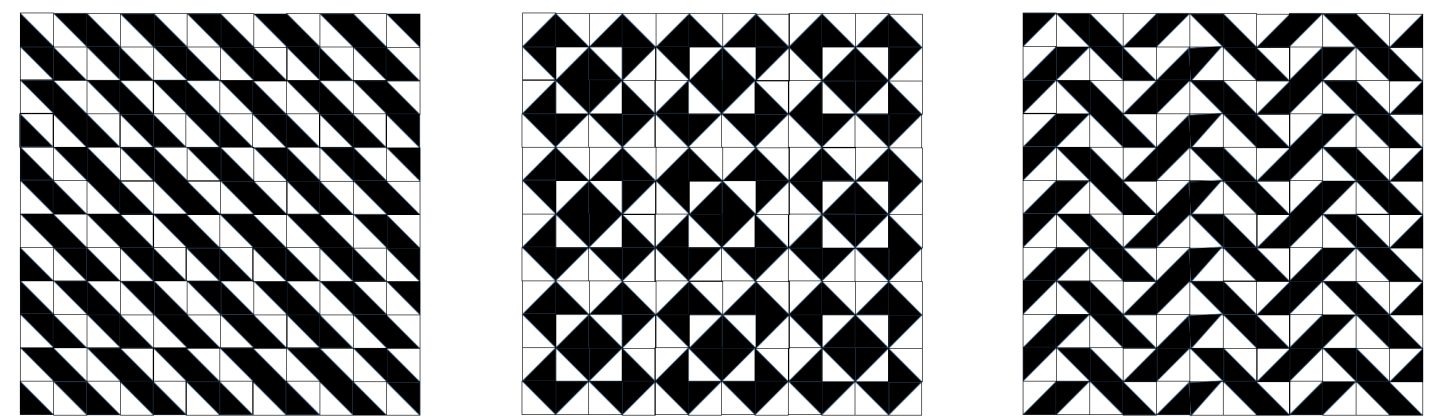

Figure 5. Wallpaper patterns constructed with square Truchet tiles, as part of a $12 \times 12$ tiling, published in 1704 .

configurations. Truchet also counted the possible combinations of two adjacent tiles and reduced these counts considering equivalence by certain isometric transformations.

Father Truchet, having been asked by Louis XIV to look after his gardens and canals, did not continue his studies on paving. Another French priest, Father Dominique Doüat (born in 1681) resumed this study and published a book in 1722, Méthode pour faire une infinité de dessins, with interesting $12 \times 12$ and $25 \times 25$ rosettes and wallpaper patterns $[3$. Figure 6 shows three $12 \times 12$ of those rosettes. Father Doüat also considered the enumeration of all possible configurations made up of three and four adjacent tiles.
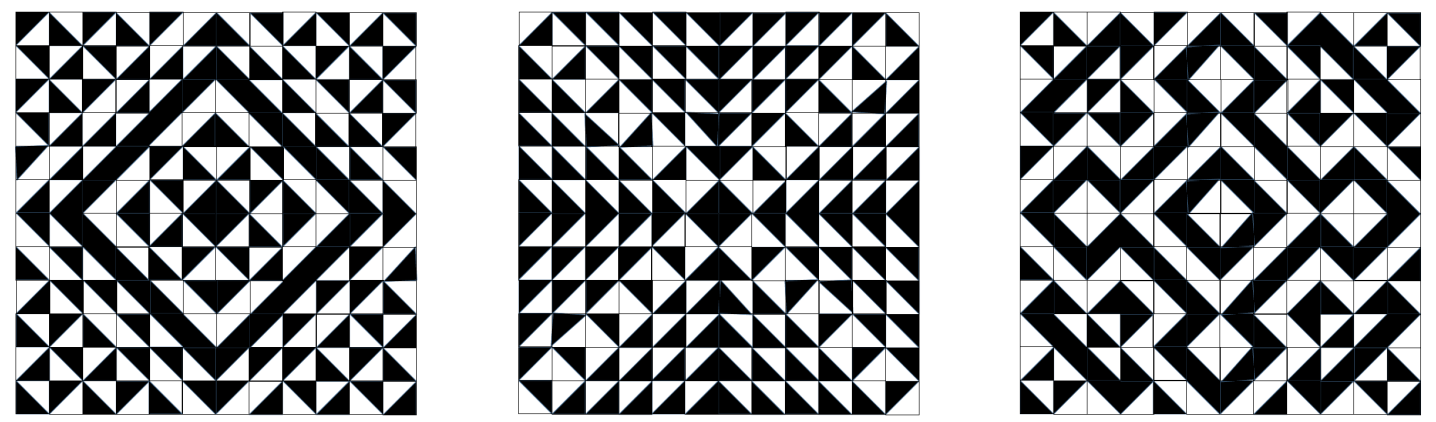

Figure 6. Rosettes constructed with square Truchet tiles, as part of a $12 \times 12$ square tiling, published in 1722 .

A question that naturally arises from the observation of Truchet and Doüat's work is "How many distinct rosettes or patterns can be created within a frame of a given size?" Indeed, in 2006 Eric Lord and S. Ranganathan already posed this challenge which we believe still remains to be answered: "An interesting exercise in combinatorics would be the enumeration and classification 
of Truchet patterns with a given symmetry and given number of tiles per unit cell" [7. This paper is a first contribution towards answering this question, by enumerating and classifying Truchet rosettes. By Truchet rosette we mean any $m \times n$ rectangular array of Truchet tiles.

Nowadays, what is commonly known as a Truchet tile is the square version of the tile, However, since in ornamental art the non-square version $\square$ is also common, we consider the slightly more general form of a Truchet tile - a rectangle divided by a diagonal line into two colored parts - including both square and non-square versions of the tile.

Considering the boundary fixed, each Truchet tile has 4 isometric positions. This is true for both the square and the non-square versions:

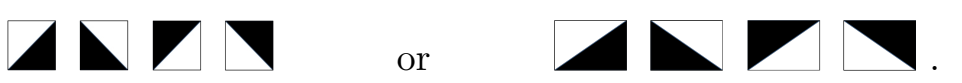

Therefore, an $m \times n$ Truchet rosette with $m, n \in \mathbb{N}$ has $4^{m n}$ possible configurations, although some will be congruent (isometric). Figure 7 shows some examples of symmetric $4 \times 4$ rosettes, using a square Truchet tile with added texture. Figure 8 shows two further examples of Truchet rosettes with square tiles, this time asymmetric $4 \times 6$, chosen from a set of randomly generated rosettes and freely colored.
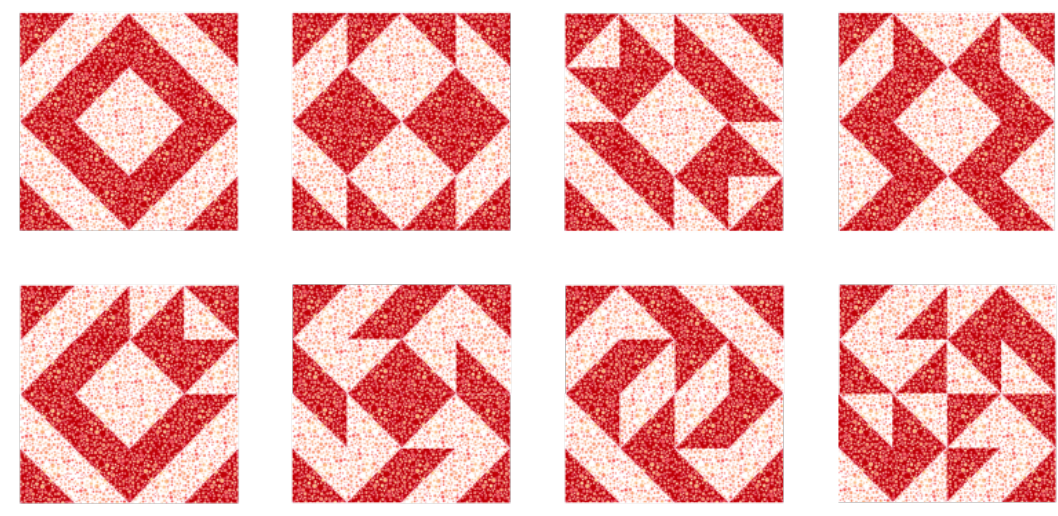

Figure 7. Rosettes $4 \times 4$ with symmetry groups (up to bottom, left to right): $D_{4}, D_{2}, D_{2}, D_{1}, D_{1}, C_{4}, C_{2}$ and $C_{2}$.
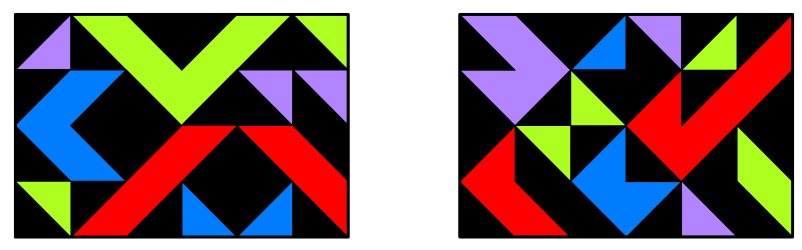

Figure 8. Randomly generated Truchet rosettes $4 \times 6$ with symmetry group $C_{1}$.

Rosettes made up of rectangular arrays of Truchet tiles are bounded by a rectangle (possibly a square) and can only have the symmetry groups exemplified in Figures 7 and $8: D_{4}, C_{4}, D_{2}$, $C_{2}, D_{1}$ and $C_{1}$. The orders of these groups are: $\left|D_{4}\right|=8,\left|C_{4}\right|=\left|D_{2}\right|=4,\left|C_{2}\right|=\left|D_{1}\right|=2$ and $\left|C_{1}\right|=1$. The largest of these groups, $D_{4}$, may be generated by a $90^{\circ}$ rotation $r$ and a reflection $f$. The complete subgroup lattice of $D_{4}$ is given in Figure 9. Note that, in the lattice, groups $D_{2}$ and $D_{1}$ are subdivided according to the reflection axes (either parallel to the sides of the square or along the diagonals); for example, the two central rosettes of the first row of Figure 7 have subgroups $D_{2}^{s}$ and $D_{2}^{d}$, respectively. This distinction will be useful in the next sections.

Let $G$ be a symmetry group. We denote the number of distinct rosettes in an $m \times n$ rectangle with symmetry group $G$, by $N(G, m, n)$ (congruent configurations count only once). Similarly, $M(G, m, n)$ denotes the total number of configurations, counting congruent ones. Since within each symmetry group $G$ congruency occurs in groups of fixed size, $M(G, m, n)$ overcounts 


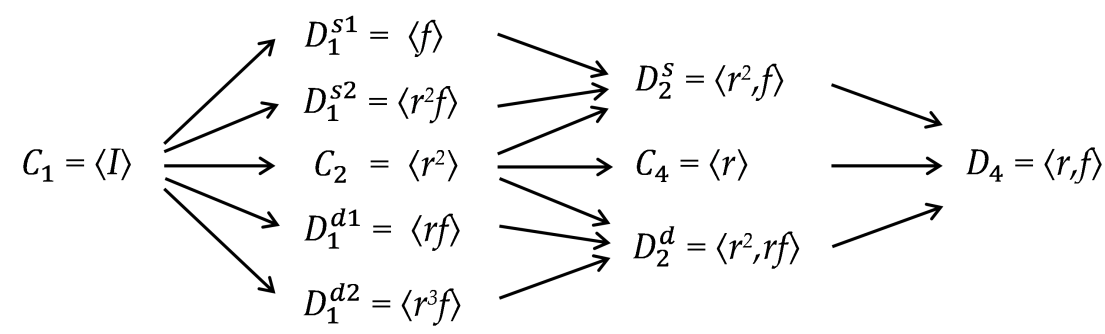

Figure 9. Subgroup lattice of $D_{4} . A \longrightarrow B$ means $A$ is a subgroup of $B ; I$ is the identity, $f$ is a vertical reflection and $r$ a $90^{\circ}$ clockwise rotation; the superscripts of subgroups $D_{n}$ identify the reflection symmetries: $s, s 1$ and $s 2$ stand for reflections parallel to both or one of the sides of the square and $d, d 1$ and $d 2$ stand for reflections along both or one of the diagonals.

$N(G, m, n)$ in a predictable way. For example, for $1 \times 2$ rosettes the total number of configurations is $4^{2}=16$ which can be split into three sets: $C_{2}, D_{1}$ and $C_{1}$ rosettes. In this case we have $N\left(D_{1}, 1,2\right)=N\left(C_{2}, 1,2\right)=N\left(C_{1}, 1,2\right)=2, M\left(D_{1}, 1,2\right)=M\left(C_{2}, 1,2\right)=4$ and $M\left(C_{1}, 1,2\right)=8$ as can be seen in Figure 10. Congruencies occur in groups of two for $C_{2}$ and $D_{1}$ and in groups of four for $C_{1}$.
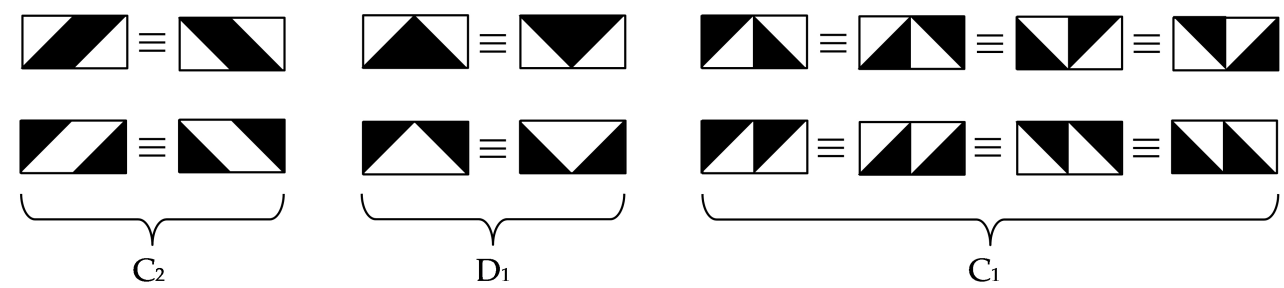

Figure 10. Rosettes $1 \times 2$ of square Truchet tiles.

From Figure 10 we can see that within each symmetry group the rosettes appear in congruency groups, all of the same size. The number of congruent rosettes is given by the ratio between the order of the bounding figure (in this case a rectangle, $\left|D_{2}\right|=4$ ) and the order of the considered group. For rosettes with a square boundary and consisting of a square array of tiles, like in Figure 7, this number is given by the ratio between the order of the symmetry group of the square, $\left|D_{4}\right|=8$, and the order of the considered group.

In this paper, we consider all possible Truchet rosettes (rectangular arrays of Truchet tiles, using either the square or the non-square version of the tile) and determine the total number of distinct rosettes for each array dimension $m \times n$, apart from an isometric transformation, $N(\cdot, m, n)$. We do this by finding this number for each possible symmetry group $G$ and adding up. To achieve this goal we start by considering the total number of configurations in each symmetry group, including congruent ones, $M(G, m, n)$, and then obtain $N(G, m, n)$ dividing the former by the number of congruent copies. As noted above, the number of congruent copies is given by the ratio between the order of the largest symmetry group possible (either that of a square or that of a non-square rectangle) and the order of the considered group.

When $m=n$ the counts differ depending on whether the tile is square or non-square. As a consequence, there is a need to distinguish the $n \times n$ square array of square tiles from all the other situations. For that purpose we shall denote by $M^{*}(G, n, n)$ and $N^{*}(G, n, n)$ the counts referring to these cases ( $n \times n$ arrays of square tiles). This way, $M(G, m, n)$ and $N(G, m, n)$ with $m=n$ only refer to arrays of non-square tiles, whereas in all other situations $M(G, m, n)$ and $N(G, m, n)$ refer to arrays of square or non-square tiles.

To count the possible configurations of rectangular Truchet tiled rosettes, $m \times n, m, n \in \mathbb{N}$, we need to consider five separate cases described in Sections 2 to 6:

Section 2: Square rosettes $n \times n$ (of square tiles) with $n$ even;

Section 3: $\quad$ Square rosettes $n \times n$ (of square tiles) with $n$ odd; 
Section 4: Rectangular rosettes $m \times n$ (of either square or non-square tiles) with both $m$ and $n$ even; (In the case $m=n$ the tile must be non-square since if it is square we fall in the first situation - Section 2.)

Section 5: Rectangular rosettes $m \times n$ (of either square or non-square tiles) with $m$ odd and $n$ even;

Section 6: Rectangular rosettes $m \times n$ with both $m$ and $n$ odd; (In the case $m=n$ the tile must be non-square otherwise we fall in the second situation - Section 3.)

The expressions providing the counts obtained throughout Sections 2 to 6 are summarized in Section 7. These expressions are also calculated for several particular values of the array dimensions, $n$ and $m$, to provide a feeling of how fast the number of configurations grows. In addition, we present a table of relative frequencies and asymptotic growth for square rosettes, in order to illustrate the probabilities of obtaining each particular group of symmetry when picking configurations at random.

In the last section of this paper we shall present some practical applications of Truchet rosettes consisting of several paper square flexagons made up of Truchet rosettes.

\section{Square rosettes, $n \times n$ with $\mathrm{n}$ even, $n=2 k, k \in \mathbb{N}$}

In this section and in Section 3 we consider only square arrays of square Truchet tiles.

Square rosettes obtained by tiling of an $n \times n$ array, can only be made up of square Truchet tiles. Square rosettes can also be obtained through $m \times n$ non-square arrays of non-square tiles with a convenient choice of the tile dimensions. For instance, we may have rectangular tiles with one side double the other and obtain a square rosette by joining two such tiles. However, these rosettes cannot have all the symmetries of a square (they cannot have the rotational symmetries of $\pm 90^{\circ}$ nor any of the reflection symmetries over the diagonals). These cases will be addresses later in Sections 4 to 6.

Restricting ourselves to square rosettes of square tiles, recall that these may have any of the six symmetry subgroup types $D_{4}, C_{4}, D_{2}, C_{2}, D_{1}$ and $C_{1}$. This happens because the symmetry group of a square is $D_{4}$ and its subgroups follow the lattice in Figure 9 .

Rosettes with symmetry group $D_{4}$ are left invariant by all eight symmetries of the square and there are no congruent copies within this group. On the other hand, $D_{2}$ rosettes (in a square) are left invariant only by four of the square symmetries (two reflections over perpendicular lines, the half turn rotation and the identity) and the other four symmetries produce a congruent copy which does not overlap. The same happens with $C_{4}$ rosettes. For both these subgroup types the rosettes appear in groups of two congruent copies each. For $C_{2}$ and $D_{1}$ rosettes (in a square), they only share two symmetries with the square. Therefore each rosette has four congruent copies which do not overlap. Finally, $C_{1}$ rosettes have eight non-overlapping congruent copies since they do not possess any symmetry apart from the identity. As mentioned in the introduction, for square rosettes the number of congruent copies within a group $G$ is given by $8 /|G|$. These properties will be extensively used throughout the present and the next sections.

In this section we consider even sided $n \times n$ rosettes, $n=2 k, k \in \mathbb{N}$.

\section{$2.1 N^{*}\left(D_{4}, 2 k, 2 k\right)$}

Rosettes with symmetry group $D_{4}$ can be generated by taking right triangles and reflecting them over the four reflection axes of symmetry of the square, as suggested in Figure 11 (left side).

By considering all the possible arrangements for the triangle we obtain all the possible $D_{4}$ rosettes. These rosettes are all distinct, $N^{*}\left(D_{4}, 2 k, 2 k\right)=M^{*}\left(D_{4}, 2 k, 2 k\right)$. There are $2^{k^{2}}$ distinct triangles. To obtain this result, note that (see the triangle on the right of Figure 11) each full 

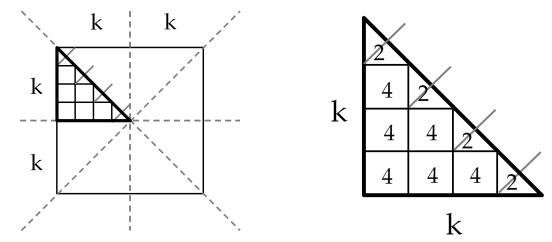

Figure 11. Generating square $D_{4}$ rosettes, $n \times n, n$ even.

tile of the triangle has 4 possible positions, while the $k$ tiles along the diagonal only have 2 positions, since in order to reflect along the diagonal of the rosette the tile must be divided perpendicularly to it (grey lines). So, the total number of configurations for the triangle is given by $2^{k} \times 4^{T_{k-1}}=2^{k} \times 4^{\frac{k(k-1)}{2}}=2^{k^{2}}$, where $T_{n}=1+2+3+\ldots+n=\frac{(n+1) n}{2}$ is the triangular number of order $n$. Therefore,

$$
M^{*}\left(D_{4}, 2 k, 2 k\right)=N^{*}\left(D_{4}, 2 k, 2 k\right)=2^{k^{2}} .
$$

Examples: For $n=2$ we have $M^{*}\left(D_{4}, 2,2\right)=N^{*}\left(D_{4}, 2,2\right)=2$ and these rosettes are illustrated in Figure 12 for $n=4$ we have $M^{*}\left(D_{4}, 4,4\right)=N^{*}\left(D_{4}, 4,4\right)=16$ and these rosettes are illustrated in Figure 13. Note that in both figures half the rosettes are color reversals of the others.

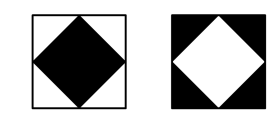

Figure 12. All possible $D_{4}$ square rosettes, $2 \times 2$.

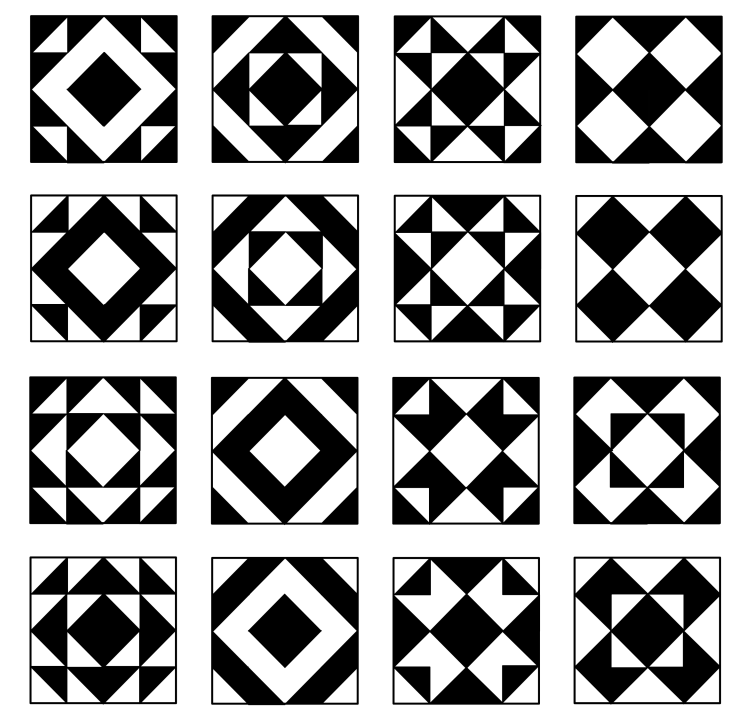

Figure 13. All possible $D_{4}$ square rosettes, $4 \times 4$.

\section{$2.2 N^{*}\left(C_{4}, 2 k, 2 k\right)$}

A $C_{4}$ rosette can be generated by taking a $k \times k$ square and rotating it multiples of a right angle around one of its corners, as suggested in Figure 14. 


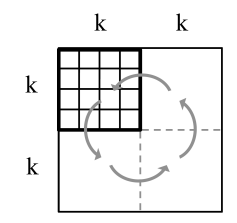

Figure 14. Generating square $C_{4}$ rosettes, $n \times n, n$ even.

By considering all the possible arrangements for the smaller square, $4^{k^{2}}$, we obtain all the possible $C_{4}$ rosettes but the procedure also generates all $D_{4}$ rosettes. Therefore $M^{*}\left(C_{4}, 2 k, 2 k\right)=$ $4^{k^{2}}-M^{*}\left(D_{4}, 2 k, 2 k\right)$. The $C_{4}$ rosettes obtained in this way are congruent in groups of two. These congruent pairs are related by a reflection along either one of the diagonals. Hence,

$$
\begin{aligned}
M^{*}\left(C_{4}, 2 k, 2 k\right) & =2^{2 k^{2}}-2^{k^{2}} \\
N^{*}\left(C_{4}, 2 k, 2 k\right) & =2^{2 k^{2}-1}-2^{k^{2}-1} .
\end{aligned}
$$

Examples: For $n=2$ we have $M^{*}\left(C_{4}, 2,2\right)=2$ and $N^{*}\left(C_{4}, 2,2\right)=1$, as illustrated in Figure 15. For $n=4$ we have $M^{*}\left(C_{4}, 4,4\right)=240$ and $N^{*}\left(C_{4}, 4,4\right)=120$ which we shall not illustrate given the large number of configurations.

\section{$\mathbf{X}=\mathbf{X}$}

Figure 15. All possible $C_{4}$ square rosettes, $2 \times 2$.

\section{$2.3 \quad N^{*}\left(D_{2}, 2 k, 2 k\right)$}

Rosettes with symmetry group $D_{2}$ may either have the reflection axes parallel to the sides of the square $\left(D_{2}^{s}\right)$ or along the diagonals $\left(D_{2}^{d}\right)$ (see Figure 9). In the first case they can be generated by reflecting $k \times k$ squares over the axes and in the second case by reflecting right triangles over the diagonals, as suggested in Figure 16.
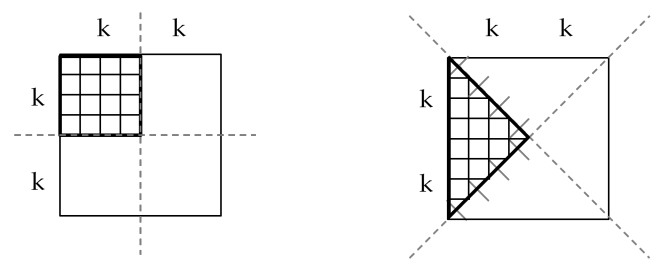

Figure 16. Generating square $D_{2}$ rosettes, $n \times n, n$ even.

In both cases the total number of generating configurations (the $k \times k$ squares or the right triangles with the same area) is $4^{k^{2}}$ (to obtain this count in the case of the right triangles we follow the same arguments as in subsection 2.1 above). Each case produces all $D_{4}$ rosettes and half of the possible $D_{2}$ ( $D_{2}^{s}$ for the first procedure and $D_{2}^{d}$ for the second). To obtain all the $D_{2}$ rosettes we must join both cases, after removing the $D_{4}$ rosettes, so $M^{*}\left(D_{2}, 2 k, 2 k\right)=$ $2 \times\left(4^{k^{2}}-M^{*}\left(D_{4}, 2 k, 2 k\right)\right)$. Since the $D_{2}$ rosettes are congruent in groups of two, we obtain:

$$
\begin{aligned}
M^{*}\left(D_{2}, 2 k, 2 k\right) & =2^{2 k^{2}+1}-2^{k^{2}+1} \\
N^{*}\left(D_{2}, 2 k, 2 k\right) & =2^{2 k^{2}}-2^{k^{2}} .
\end{aligned}
$$


Example: For $n=2$ we have $M^{*}\left(D_{2}, 2,2\right)=4$ and $N^{*}\left(D_{2}, 2,2\right)=2$, as illustrated in Figure 17 .

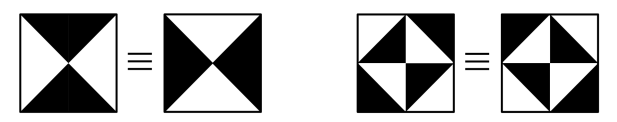

Figure 17. All possible $D_{2}$ square rosettes, $2 \times 2$.

\section{$2.4 N^{*}\left(C_{2}, 2 k, 2 k\right)$}

A $C_{2}$ rosette can be generated by taking a $2 k \times k$ rectangle and rotating it half a turn, as suggested in Figure 18 .

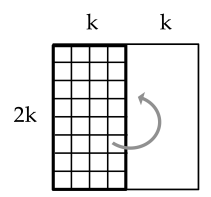

Figure 18. Generating square $C_{2}$ rosettes, $n \times n, n$ even.

By considering all the possible arrangements for the rectangle we obtain all the possible $C_{2}, D_{2}$, $C_{4}$ and $D_{4}$ rosettes. The total number of possible rectangles is $4^{2 k^{2}}$. Therefore $M^{*}\left(C_{2}, 2 k, 2 k\right)=$ $4^{2 k^{2}}-M^{*}\left(D_{2}, 2 k, 2 k\right)-M^{*}\left(C_{4}, 2 k, 2 k\right)-M^{*}\left(D_{4}, 2 k, 2 k\right)$. The $C_{2}$ rosettes obtained in this way are congruent in groups of four. As a result,

$$
\begin{aligned}
M^{*}\left(C_{2}, 2 k, 2 k\right) & =2^{4 k^{2}}-3 \times 2^{2 k^{2}}+2^{k^{2}+1} \\
N^{*}\left(C_{2}, 2 k, 2 k\right) & =2^{4 k^{2}-2}-3 \times 2^{2 k^{2}-2}+2^{k^{2}-1} .
\end{aligned}
$$

Example: For $n=2$ we have $M\left(C_{2}, 2,2\right)=8$ and $N^{*}\left(C_{2}, 2,2\right)=2$, as illustrated in Figure [19.

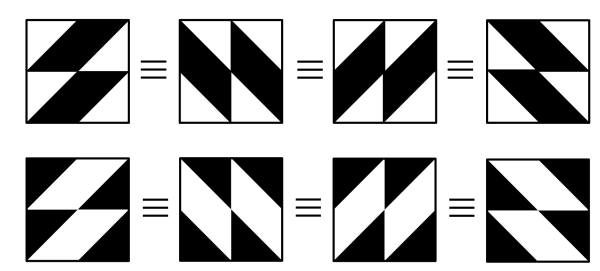

Figure 19. All possible $C_{2}$ square rosettes, $2 \times 2$.

\section{$2.5 N^{*}\left(D_{1}, 2 k, 2 k\right)$}

Rosettes with symmetry group $D_{1}$ may have the reflection axis parallel to one of the sides of the square $\left(D_{1}^{s 1}\right.$ or $\left.D_{1}^{s 2}\right)$ or as one of its diagonals $\left(D_{1}^{d 1}\right.$ or $\left.D_{1}^{d 2}\right)$ (see Figure 9). They can be generated by: reflecting a $k \times 2 k$ rectangle over the vertical axis; reflecting a $2 k \times k$ rectangle over the horizontal axis; reflecting a right triangle (half the square) over each of the diagonals, as suggested in Figure 20.

The rosettes generated by the first two procedures are congruent (they differ by a right angle rotation). The same happens with the rosettes generated from the last two procedures. In all 

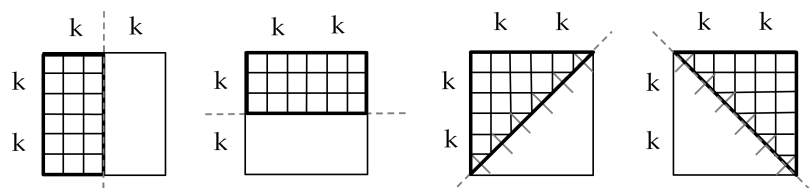

Figure 20. Generating square $D_{1}$ rosettes, $n \times n, n$ even.

cases the generated rosettes can be $D_{1}, D_{2}$, or $D_{4}$ rosettes. In each of the four cases the total number of generating configurations (the rectangles or the right triangles) is $4^{2 k^{2}}$ (to obtain this count in the case of the right triangles we follow the same arguments as in subsection 2.1 above). Each case produces one quarter of the $D_{1}$ rosettes $\left(D_{1}^{s 1}, D_{1}^{s 2}, D_{1}^{d 1}, D_{1}^{d 2}\right.$, respectively), half of the possible $D_{2}$ ( $D_{2}^{s}$ for the first two and $D_{2}^{d}$ for the last two) and all $D_{4}$ (see Figure 9 ). To obtain all the $D_{1}$ rosettes we must join the four cases, after removing the $D_{2}$ and the $D_{4}$ rosettes. Hence,

$$
M^{*}\left(D_{1}, 2 k, 2 k\right)=4 \times\left(4^{2 k^{2}}-M^{*}\left(D_{2}, 2 k, 2 k\right) / 2-M^{*}\left(D_{4}, 2 k, 2 k\right)\right) .
$$

These rosettes are congruent in groups of four. As a result we obtain,

$$
\begin{aligned}
M^{*}\left(D_{1}, 2 k, 2 k\right) & =2^{4 k^{2}+2}-2^{2 k^{2}+2} \\
N^{*}\left(D_{1}, 2 k, 2 k\right) & =2^{4 k^{2}}-2^{2 k^{2}} .
\end{aligned}
$$

Example: For $n=2$ we have $M^{*}\left(D_{1}, 2,2\right)=48$ and $N^{*}\left(D_{1}, 2,2\right)=12$. We shall only represent the 12 distinct rosettes (apart from an isometric transformation) in Figure 21.

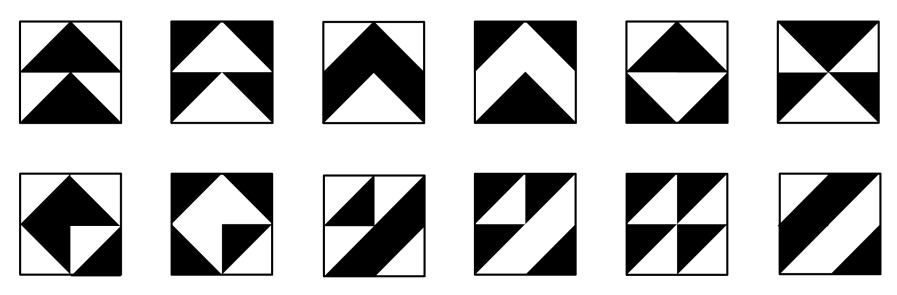

Figure 21. All distinct $D_{1}$ square rosettes, $2 \times 2$.

\section{$2.6 N^{*}\left(C_{1}, 2 k, 2 k\right)$}

Rosettes from symmetry group $C_{1}$ are all the remaining rosettes not considered so far. They are congruent in groups of eight. The total number of $n \times n, n=2 k$, square rosettes (including congruent copies) is $4^{4 k^{2}}$. Hence $M^{*}\left(C_{1}, 2 k, 2 k\right)=4^{4 k^{2}}-M^{*}\left(D_{1}, 2 k, 2 k\right)-M^{*}\left(C_{2}, 2 k, 2 k\right)-$ $M^{*}\left(D_{2}, 2 k, 2 k\right)-M^{*}\left(C_{4}, 2 k, 2 k\right)-M^{*}\left(D_{4}, 2 k, 2 k\right)$,

resulting in the following expressions

$$
\begin{aligned}
M^{*}\left(C_{1}, 2 k, 2 k\right) & =2^{8 k^{2}}-5 \times 2^{4 k^{2}}+2^{2 k^{2}+2} \\
N^{*}\left(C_{1}, 2 k, 2 k\right) & =2^{8 k^{2}-3}-5 \times 2^{4 k^{2}-3}+2^{2 k^{2}-1} .
\end{aligned}
$$

Example: For $n=2$ we have $M^{*}\left(C_{1}, 2,2\right)=192$ and $N^{*}\left(C_{1}, 2,2\right)=24$. We shall only represent the 24 distinct rosettes (apart from an isometric transformation) in Figure 22. 


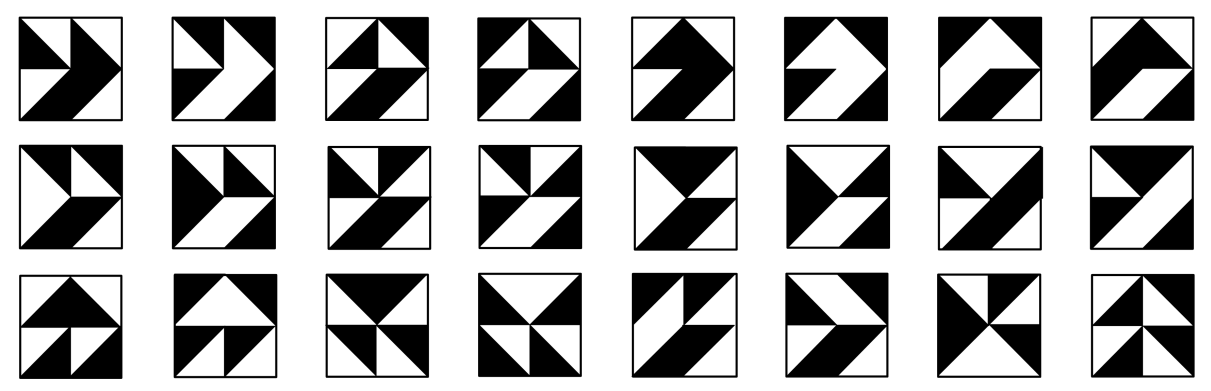

Figure 22. All distinct $C_{1}$ square rosettes, $2 \times 2$.

\subsection{Total number of distinct rosettes, $N^{*}(\cdot, 2 k, 2 k)$}

Let $T=\left\{D_{4}, C_{4}, D_{2}, C_{2}, D_{1}, C_{1}\right\}$. The total number of distinct square rosettes, $n \times n$ with $n=2 k$, is given by

$$
\begin{aligned}
N^{*}(\cdot, 2 k, 2 k) & =\sum_{G \in T} N^{*}(G, 2 k, 2 k) \\
& =2^{8 k^{2}-3}+5 \times 2^{4 k^{2}-3}+2^{2 k^{2}-2} .
\end{aligned}
$$

In the case of $2 \times 2$ rosettes we have a total of $N^{*}(\cdot, 2,2)=2+1+2+2+12+24=43$ rosettes which have been illustrated in the preceding figures.

\section{Square rosettes $n \times n$ with $\mathrm{n}$ odd, $n=2 k-1, k \in \mathbb{N}$}

Recall that square rosettes $n \times n$, can only be made up of Truchet square tiles. When the number of tiles is odd, the center of the rosette is the center of a tile and not one of its vertices. This means that at most one reflection axis is possible (through one of the diagonals) and no nontrivial rotational symmetries are possible. Therefore these rosettes can only have the symmetry groups $D_{1}\left(D_{1}^{d 1}\right.$ or $\left.D_{1}^{d 2}\right)$ and $C_{1}$. Examples of $D_{1}$ rosettes are given in Figure 24.

\section{$3.1 N^{*}\left(D_{1}, 2 k-1,2 k-1\right)$}

An odd sized $D_{1}$ square rosette can be generated by reflecting a right triangle (half of the square) over one of the diagonals, as suggested in Figure 23.
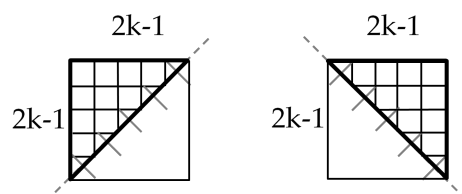

Figure 23. Generating square $D_{1}$ rosettes, $n \times n, n$ odd.

The two sets of rosettes generated by these two procedures are congruent (they differ by a rotation of 90 degrees). In each case the total number of generating configurations (right triangles) is $4^{n^{2} / 2}=2^{n^{2}}$, where $n=2 k-1$. All the generated rosettes are $D_{1}$. Within each set of generated rosettes, they are congruent in groups of two (by a reflection over the other diagonal). By joining the two sets, we obtain all the possible configurations of $D_{1}$ rosettes, and these are 
congruent in groups of four. Therefore,

$$
\begin{aligned}
& M^{*}\left(D_{1}, n, n\right)=2^{n^{2}+1} \\
& N^{*}\left(D_{1}, n, n\right)=2^{n^{2}-1},
\end{aligned}
$$

for $n=2 k-1$, with $k \in \mathbb{N}$.

Example: For $n=1$ we have $M^{*}\left(D_{1}, 1,1\right)=4$ and $N^{*}\left(D_{1}, 1,1\right)=1$. This corresponds to the trivial case of a single tile rosette. For $n=3$ we have $M^{*}\left(D_{1}, 3,3\right)=1024$ and $N^{*}\left(D_{1}, 3,3\right)=256$. Three of these rosettes are illustrated in Figure 24.

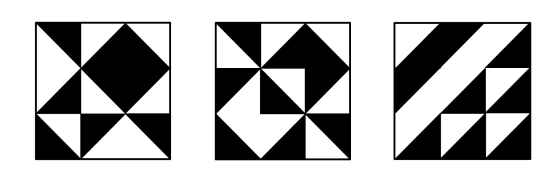

Figure 24. Examples of $D_{1}$ square rosettes, $3 \times 3$.

\section{$3.2 \quad N^{*}\left(C_{1}, 2 k-1,2 k-1\right)$}

Rosettes with symmetry group $C_{1}$ are all the other rosettes. They are congruent in groups of eight. The total number of square rosettes $n \times n$, with $n=2 k-1$ (including congruent copies), is $4^{n^{2}}$. Therefore $M^{*}\left(C_{1}, n, n\right)=4^{n^{2}}-M^{*}\left(D_{1}, n, n\right)$, resulting in the following expressions:

$$
\begin{aligned}
M^{*}\left(C_{1}, n, n\right) & =2^{2 n^{2}}-2^{n^{2}+1} \\
N^{*}\left(C_{1}, n, n\right) & =2^{2 n^{2}-3}-2^{n^{2}-2},
\end{aligned}
$$

for $n=2 k-1$, with $k \in \mathbb{N}$.

\subsection{Total number of distinct rosettes, $N^{*}(\cdot, 2 k-1,2 k-1)$}

The total number of distinct rosettes is given by

$$
N^{*}(\cdot, n, n)=N^{*}\left(D_{1}, n, n\right)+N^{*}\left(C_{1}, n, n\right)
$$

resulting in

$$
N^{*}(\cdot, n, n)=2^{2 n^{2}-3}+2^{n^{2}-2},
$$

for $n=2 k-1$, with $k \in \mathbb{N}$.

Therefore, there is only one distinct $1 \times 1$ square rosette but a total of $N^{*}(\cdot, 3,3)=32896$ distinct $3 \times 3$ square rosettes.

\section{Rectangular rosettes $m \times n$ with both $m$ and $n$ even, $m=2 j, n=2 k, j, k \in \mathbb{N}$}

In the next sections we consider general rectangular rosettes which can be built by tiling an $m \times n$ rectangular array with either square or non-square Truchet tiles. Unfortunately, the case $n=m$ requires different approaches depending on whether the tile is square or non-square. Square arrays $(n \times n)$ with square tiles have been addressed in the previous two sections. Therefore, hereafter we consider rectangular arrays $m \times n$ of Truchet tiles which must fall into one of the next cases: 
- if $n=m$ the tile must be non-square;

- if $n \neq m$ the tile can be either square or non square.

In other words, in all cases considered hereafter either the rosette or the tile (or both) must be a non-square rectangle. Therefore the greatest symmetry group of the rosette is that of a rectangle, $D_{2}$, which has four symmetries. This means that $D_{2}$ rosettes are left invariant by all the four symmetries of the rectangle. Rosettes with symmetry group $D_{1}$ are left invariant only by two of the rectangle symmetries and the other two symmetries produce a congruent copy which does not overlap. The same happens with $C_{2}$ rosettes. Rosettes with symmetry group $C_{1}$ have four non-overlapping congruent copies since they do not possess any symmetry apart from the identity.

We start in this section by considering $m \times n$ rectangular arrays with both $m$ and $n$ even, $m=2 j, n=2 k, j, k \in \mathbb{N}$. All such rosettes can have symmetry groups $D_{2}, C_{2}, D_{1}$ and $C_{1}$.

\section{$4.1 N\left(D_{2}, 2 j, 2 k\right)$}

Rosettes with symmetry group $D_{2}$ may only have reflection axes parallel to the sides of the rectangle $\left(D_{2}^{s}\right)$. They can be generated by reflecting $j \times k$ rectangles over the axes, as suggested in Figure 25.

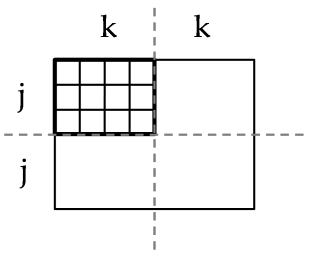

Figure 25. Generating rectangular $D_{2}$ rosettes, $m \times n, m$ and $n$ both even.

The total number of generating configurations (the $j \times k$ rectangles) is $4^{j k}$. All $D_{2}$ rosettes are generated in this way and there are no congruencies. Hence,

$$
M\left(D_{2}, 2 j, 2 k\right)=N\left(D_{2}, 2 j, 2 k\right)=2^{2 j k} .
$$

Examples: For $m=2$ and $n=4$ (square and non-square tiles) we have $N\left(D_{2}, 2,4\right)=$ $M\left(D_{2}, 2,4\right)=16$, as illustrated in Figure 26 .
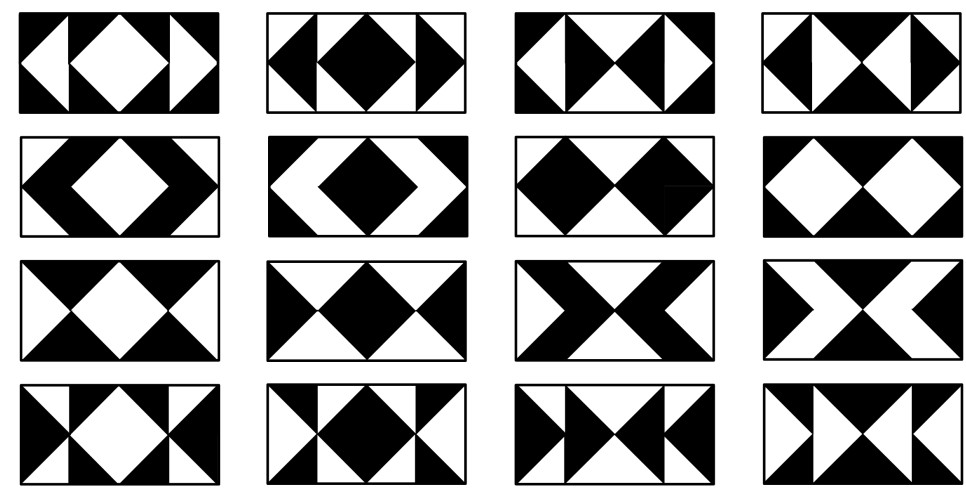

Figure 26. All distinct $D_{2}$ non-square rosettes, $2 \times 4$, with a square tile.

For $n=2$ and $m=2$ (non-square tile) we have $N\left(D_{2}, 2,2\right)=M\left(D_{2}, 2,2\right)=4$, as illustrated in Figure 27. 

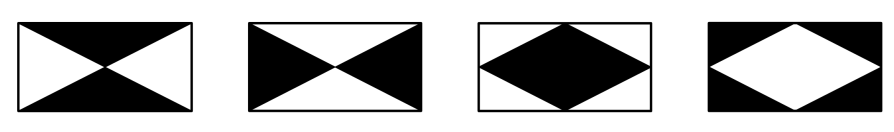

Figure 27. All distinct $D_{2}$ non-square rosettes, $2 \times 2$, with a non-square tile.

\section{$4.2 N\left(C_{2}, 2 j, 2 k\right)$}

A $C_{2}$ rosette can be generated by taking a $2 j \times k$ rectangle and rotating it half a turn, as suggested in Figure 28 .

$2 \mathrm{j}$

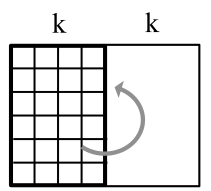

Figure 28. Generating rectangular $C_{2}$ rosettes, $m \times n, m$ and $n$ both even.

By considering all the possible arrangements for the rectangle we obtain all the possible $C_{2}$ and also $D_{2}$ rosettes. The total number of possible rectangles is $4^{2 j k}$. Therefore $M\left(C_{2}, 2 j, 2 k\right)=$ $4^{2 j k}-M\left(D_{2}, 2 j, 2 k\right)$. The $C_{2}$ rosettes obtained in this way are congruent in groups of two. Hence

$$
\begin{aligned}
M\left(C_{2}, 2 j, 2 k\right) & =2^{4 j k}-2^{2 j k} \\
N\left(C_{2}, 2 j, 2 k\right) & =2^{4 j k-1}-2^{2 j k-1}
\end{aligned}
$$

Examples: For $m=2$ and $n=2$ (non-square tile) we have $M\left(C_{2}, 2,2\right)=12$ and $N\left(C_{2}, 2,2\right)=$ 6 , as illustrated in Figure 29. For $m=2$ and $n=4$ we have $M\left(C_{2}, 2,2\right)=240$ and $N\left(C_{2}, 2,2\right)=$ 120.
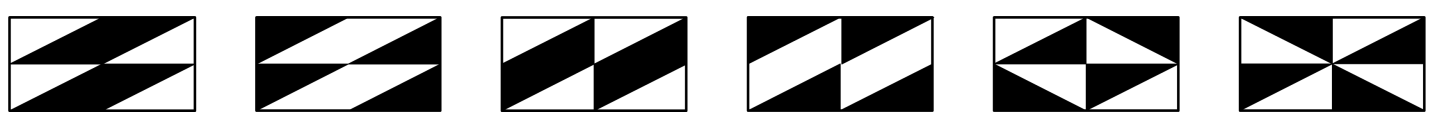

Figure 29. All distinct $C_{2}$ non-square rosettes, $2 \times 2$, with a non-square tile.

\section{$4.3 N\left(D_{1}, 2 j, 2 k\right)$}

Rosettes with symmetry group $D_{1}$ have the reflection axis parallel to one of the sides of the rectangle $\left(D_{2}^{s 1}\right.$ or $\left.D_{2}^{s 2}\right)$. They can be generated by reflecting a $2 j \times k$ rectangle over the vertical axis or by reflecting a $j \times 2 k$ rectangle over the horizontal axis, as suggested in Figure 30 .
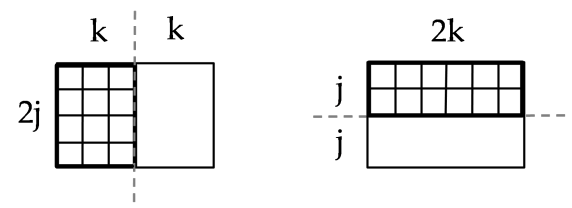

Figure 30. Generating rectangular $D_{1}$ rosettes, $m \times n, m$ and $n$ both even.

The rosettes generated by these procedures can be $D_{1}$ or $D_{2}$. The $D_{1}$ rosettes are congruent in groups of two. In each case the total number of generating configurations (the rectangles) is $4^{2 j k}$. Each case produces all $D_{2}$ rosettes, and half of the $D_{1}$ (disjoint sets). To obtain all the $D_{1}$ 
rosettes we must join the two cases, after removing the $D_{2}$ configurations. Hence, $M\left(D_{1}, 2 j, 2 k\right)=$ $2 \times\left(4^{2 j k}-M\left(D_{2}, 2 j, 2 k\right)\right)$. Therefore,

$$
\begin{aligned}
M\left(D_{1}, 2 j, 2 k\right) & =2^{4 k j+1}-2^{2 k j+1} \\
N\left(D_{1}, 2 j, 2 k\right) & =2^{4 k j}-2^{2 k j}
\end{aligned}
$$

Examples: For $n=2$ and $m=2$ (non-square tile) we have $M\left(D_{1}, 2,2\right)=24$ and $N\left(D_{1}, 2,2\right)=$ 12, as illustrated in Figure 31

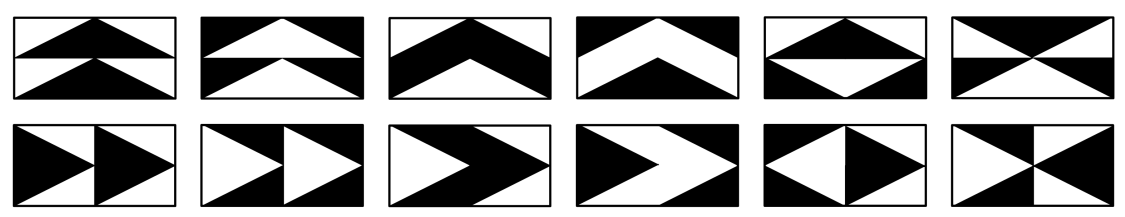

Figure 31. All distinct $D_{1}$ non-square rosettes, $2 \times 2$, with a non-square tile.

\section{$4.4 N\left(C_{1}, 2 j, 2 k\right)$}

Rosettes with symmetry group $C_{1}$ are all the remaining rosettes not considered so far. They are congruent in groups of four. The total number of $n \times m$ rosettes (including congruent copies) is $4^{4 j k}$. Hence $M\left(C_{1}, 2 j, 2 k\right)=4^{4 j k}-M\left(D_{1}, 2 j, 2 k\right)-M\left(C_{2}, 2 j, 2 k\right)-M\left(D_{2}, 2 j, 2 k\right)$, resulting in the following expressions

$$
\begin{aligned}
& M\left(C_{1}, 2 j, 2 k\right)=2^{8 j k}-3 \times 2^{4 j k}+2^{2 j k+1} \\
& N\left(C_{1}, 2 j, 2 k\right)=2^{8 j k-2}-3 \times 2^{4 j k-2}+2^{2 j k-1}
\end{aligned}
$$

Example: For $m=2$ and $n=2$ (non-square tile) we have $M\left(C_{1}, 2,2\right)=216$ and $N\left(C_{1}, 2,2\right)=$ 54 . We shall not represent these cases but twenty-four rosettes are obtained by stretching the twenty-four $C_{1}$ square rosettes (see Figure 22 horizontally, and an additional twenty-four rosettes arise from stretching vertically, instead. The remaining six rosettes are stretched versions of the six $D_{1}$ square rosettes, $2 \times 2$, with reflection axis along the diagonal (see second row of Figure 21), in which case stretching in either direction gives the same result).

\subsection{Total number of distinct rosettes, $N(\cdot, 2 j, 2 k)$}

The total number of distinct rosettes is given by

$$
\begin{aligned}
N(\cdot, 2 j, 2 k) & =N\left(D_{2}, 2 j, 2 k\right)+N\left(C_{2}, 2 j, 2 k\right)+N\left(D_{1}, 2 j, 2 k\right)+N\left(C_{1}, 2 j, 2 k\right) \\
& =2^{8 j k-2}+3 \times 2^{4 j k-2}
\end{aligned}
$$

\section{Rectangular rosettes $m \times n$ with $m$ odd and $n$ even,} $m=2 j-1, n=2 k, k, j \in \mathbb{N}$

These rosettes have their center on the mid-point of one of the sides of the tile. As a consequence, there is only one possible reflection axis and it passes through that side. Also, rotational symmetries can only be of order 2 . Hence, these rosettes can only have symmetry groups $C_{2}, D_{1}$ and $C_{1}$. As an example see Figure 10 containing all $1 \times 2$ rosettes and their symmetry groups. 


\section{$5.1 \quad N\left(C_{2}, m, 2 k\right), m=2 j-1$}

Like in previous sections, $C_{2}$ rosettes can be generated by taking a rectangle (this time $m \times k$ ) and rotating it half a turn. By considering all the possible arrangements for the rectangle (there are $4^{m k}$ such arrangements) we obtain all the possible $C_{2}$ rosettes. These are congruent in groups of two. Hence,

$$
\begin{aligned}
& M\left(C_{2}, m, 2 k\right)=2^{2 m k} \\
& N\left(C_{2}, m, 2 k\right)=2^{2 m k-1},
\end{aligned}
$$

for $m=2 j-1$, with $j \in \mathbb{N}$.

\section{$5.2 N\left(D_{1}, m, 2 k\right), m=2 j-1$}

Rosettes with symmetry group $D_{1}$ have the reflection axis parallel to one of the sides of the rectangle $\left(D_{2}^{s 1}\right.$ or $\left.D_{2}^{s 2}\right)$. As before, they can be generated by reflecting a rectangle (this time $m \times k)$. The rosettes generated are all $D_{1}$ and congruent in groups of two. The total number of generating configurations (the rectangles) is $4^{2 j k}$. Hence,

$$
\begin{aligned}
M\left(D_{1}, m, 2 k\right) & =2^{2 m k} \\
N\left(D_{1}, m, 2 k\right) & =2^{2 m k-1},
\end{aligned}
$$

for $m=2 j-1$, with $j \in \mathbb{N}$.

\section{$5.3 N\left(C_{1}, m, 2 k\right), m=2 j-1$}

Rosettes with symmetry group $C_{1}$ are all the remaining rosettes not considered so far. They are congruent in groups of four. The total number of $n \times m$ rectangular rosettes (including congruent copies $)$ is $4^{2 m k}$. Hence $M\left(C_{1}, m, 2 k\right)=4^{2 m k}-M\left(D_{1}, m, 2 k\right)-M\left(C_{2}, m, 2 k\right)$, resulting in the following expressions

$$
\begin{aligned}
& M\left(C_{1}, m, 2 k\right)=2^{4 m k}-2^{2 m k+1} \\
& N\left(C_{1}, m, 2 k\right)=2^{4 m k-2}-2^{2 m k-1},
\end{aligned}
$$

for $m=2 j-1$, with $j \in \mathbb{N}$.

\subsection{Total number of distinct rosettes, $N(\cdot, m, 2 k), m=2 j-1$}

The total number of distinct rosettes is given by

$$
\begin{aligned}
N(\cdot, m, 2 k) & =N\left(C_{2}, m, 2 k\right)+N\left(D_{1}, m, 2 k\right)+N\left(C_{1}, m, 2 k\right) \\
& =2^{4 m k-2}+2^{2 m k-1}
\end{aligned}
$$

for $m=2 j-1$, with $j \in \mathbb{N}$. 


\section{Rectangular rosettes $m \times n$ with both $m$ and $n$ odd, $m=2 j-1, n=2 k-1, j, k \in \mathbb{N}$}

Like the square odd-sized rosettes, these rosettes have their center on the mid-point of a tile. As a consequence, there are no possible reflection axes or rotational symmetries of order two or more (recall that either the tile or the rosette must be non-square). Hence, these rosettes can only have symmetry group $C_{1}$ and they are congruent in groups of four. The total number of $m \times n$ rectangular rosettes (including congruent copies) is $4^{m n}$. Hence,

$$
\begin{aligned}
M\left(C_{1}, m, n\right) & =2^{2 m n} \\
N\left(C_{1}, m, n\right) & =2^{2 m n-2},
\end{aligned}
$$

for $m=2 j-1$ and $n=2 k-1$, with $j, k \in \mathbb{N}$. 32 .

For $m=1$ and $n=3$ we have $M\left(C_{1}, 1,3\right)=64$ and $N\left(C_{1}, 1,3\right)=16$, as illustrated in Figure

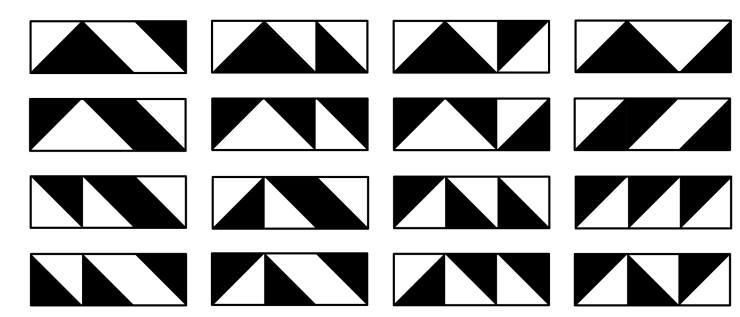

Figure 32. All distinct $C_{1}$ rosettes, $1 \times 3$, with a square tile.

\section{Counts of rosettes grow fast}

In this section, we collect all the expressions obtained in the previous sections in four tables, two for square rosettes of square tiles (counts $M^{*}$ and $N^{*}$ from Sections 2 and 3) and two for all other rectangular rosettes (counts $M$ and $N$ from Sections 4 to 6), to allow for growth analysis based on rosette size.

\begin{tabular}{|c|c|c|}
\hline \multicolumn{3}{|c|}{$\boldsymbol{M}^{*}(\boldsymbol{G}, \boldsymbol{n}, \boldsymbol{n})$} \\
\hline $\boldsymbol{G}$ & $\boldsymbol{n}$ even, $\boldsymbol{n}=\mathbf{2} \boldsymbol{k}, \boldsymbol{k} \in \mathbf{I N}$ & $\boldsymbol{n}$ odd, $\boldsymbol{n}=\mathbf{2} \boldsymbol{k}-\mathbf{1}, \boldsymbol{k} \in \mathbf{I N}$ \\
\hline \hline $\boldsymbol{D}_{\boldsymbol{4}}$ & $2^{k^{2}}$ & \\
\hline $\boldsymbol{C}_{\boldsymbol{4}}$ & $2^{2 k^{2}}-2^{k^{2}}$ & \\
\hline $\boldsymbol{D}_{\mathbf{2}}$ & $2^{2 k^{2}+1}-2^{k^{2}+1}$ & \\
\hline $\boldsymbol{C}_{\mathbf{2}}$ & $2^{4 k^{2}}-3 \times 2^{2 k^{2}}+2^{k^{2}+1}$ & $2^{n^{2}+1}$ \\
\hline $\boldsymbol{D}_{\mathbf{1}}$ & $2^{4 k^{2}+2}-2^{2 k^{2}+2}$ & $2^{2 n^{2}}-2^{n^{2}+1}$ \\
\hline $\boldsymbol{C}_{\mathbf{1}}$ & $2^{8 k^{2}}-5 \times 2^{4 k^{2}}+2^{2 k^{2}+2}$ & $4^{n^{2}}$ \\
\hline Total & $4^{n^{2}}$ & \\
\hline
\end{tabular}

Table 1. $M^{*}(G, n, n)$ for square rosettes of square tiles.

Looking at the expressions in Tables 1 through 4 , we can see that the counts grow exponentially fast as we increase the size of the rosette.

Tables 5 through 7 contain the number of rosettes for each symmetry group, for the first values of $m$ and $n$ (using the expressions in the previous tables). In each table bold numbers refer to 


\begin{tabular}{|c|c|c|}
\hline \multicolumn{3}{|c|}{$\boldsymbol{N}^{*}(\boldsymbol{G}, \boldsymbol{n}, \boldsymbol{n})$} \\
\hline $\boldsymbol{G}$ & $\boldsymbol{n}$ even, $\boldsymbol{n}=\mathbf{2} \boldsymbol{k}, \boldsymbol{k} \in \mathbf{I N}$ & $\boldsymbol{n}$ odd, $\boldsymbol{n}=\mathbf{2} \boldsymbol{k}-\mathbf{1}, \boldsymbol{k} \in \mathbf{I N}$ \\
\hline \hline $\boldsymbol{D}_{\mathbf{4}}$ & $2^{k^{2}}$ & \\
\hline $\boldsymbol{C}_{\boldsymbol{4}}$ & $2^{2 k^{2}-1}-2^{k^{2}-1}$ & \\
\hline $\boldsymbol{D}_{\mathbf{2}}$ & $2^{2 k^{2}}-2^{k^{2}}$ & \\
\hline $\boldsymbol{C}_{\mathbf{2}}$ & $2^{4 k^{2}-2}-3 \times 2^{2 k^{2}-2}+2^{k^{2}-1}$ & $2^{n^{2}-1}$ \\
\hline $\boldsymbol{D}_{\mathbf{1}}$ & $2^{4 k^{2}}-2^{2 k^{2}}$ & $2^{2 n^{2}-3}-2^{n^{2}-2}$ \\
\hline $\boldsymbol{C}_{\mathbf{1}}$ & $2^{8 k^{2}-3}-5 \times 2^{4 k^{2}-3}+2^{2 k^{2}-1}$ & $2^{2 n^{2}-3}+2^{n^{2}-2}$ \\
\hline Total & $2^{8 k^{2}-3}+5 \times 2^{4 k^{2}-3}+2^{2 k^{2}-2}$ & \\
\hline
\end{tabular}

Table 2. $N^{*}(G, n, n)$ for square rosettes of square tiles.

\begin{tabular}{|c|c|c|c|}
\hline \multicolumn{4}{|c|}{$\boldsymbol{M}(\boldsymbol{G}, \boldsymbol{m}, \boldsymbol{n})$} \\
\hline $\boldsymbol{G}$ & $\boldsymbol{m}=\mathbf{2} \boldsymbol{j}, \boldsymbol{n}=\mathbf{2} \boldsymbol{k}$ & $\boldsymbol{m}=\mathbf{2} \boldsymbol{j}-\mathbf{1}, \boldsymbol{n}=\mathbf{2} \boldsymbol{k}$ & $\boldsymbol{m}=\mathbf{2} \boldsymbol{j}, \boldsymbol{n}=\mathbf{2} \boldsymbol{k}$ \\
\hline \hline $\boldsymbol{D}_{\mathbf{2}}$ & $2^{2 j k}$ & & \\
\hline $\boldsymbol{C}_{\mathbf{2}}$ & $2^{4 j k}-2^{2 j k}$ & $2^{2 m k}$ & \\
\hline $\boldsymbol{D}_{\mathbf{1}}$ & $2^{4 k j+1}-2^{2 k j+1}$ & $2^{2 m k}$ & \\
\hline $\boldsymbol{C}_{\mathbf{1}}$ & $2^{8 j k}-3 \times 2^{4 j k}+2^{2 j k+1}$ & $2^{4 m k}-2^{2 m k+1}$ & $2^{2 m n}$ \\
\hline Total & $4^{m n}$ & $4^{m n}$ & $4^{m n}$ \\
\hline
\end{tabular}

Table 3. $M(G, m, n)$ for general rosettes, excluding square rosettes of square tiles.

\begin{tabular}{|c|c|c|c|}
\hline \multicolumn{4}{|c|}{$\boldsymbol{N}(\boldsymbol{G}, \boldsymbol{m}, \boldsymbol{n})$} \\
\hline $\boldsymbol{G}$ & $\boldsymbol{m}=\mathbf{2} \boldsymbol{j}, \boldsymbol{n}=\mathbf{2} \boldsymbol{k}$ & $\boldsymbol{m}=\mathbf{2} \boldsymbol{j}-\mathbf{1}, \boldsymbol{n}=\mathbf{2} \boldsymbol{k}$ & $\boldsymbol{m}=\mathbf{2} \boldsymbol{j}, \boldsymbol{n}=\mathbf{2} \boldsymbol{k}$ \\
\hline \hline $\boldsymbol{D}_{\mathbf{2}}$ & $2^{2 j k}$ & & \\
\hline $\boldsymbol{C}_{\mathbf{2}}$ & $2^{4 j k-1}-2^{2 j k-1}$ & $2^{2 m k-1}$ & \\
\hline $\boldsymbol{D}_{\mathbf{1}}$ & $2^{4 k j}-2^{2 k j}$ & $2^{2 m k-1}$ & \\
\hline $\boldsymbol{C}_{\mathbf{1}}$ & $2^{8 j k-2}-3 \times 2^{4 j k-2}+2^{2 j k-1}$ & $2^{4 m k-2}-2^{2 m k-1}$ & $2^{2 m n-2}$ \\
\hline Total & $2^{8 j k-2}+3 \times 2^{4 j k-2}$ & $2^{4 m k-2}+2^{2 m k-1}$ & $2^{2 m n-2}$ \\
\hline
\end{tabular}

Table 4. $N(G, m, n)$ for rectangular rosettes, excluding square rosettes of square tiles.

rosettes having been (completely) illustrated in the figures throughout the text. While for small values of $m$ and $n$ it is interesting to enumerate all the possible configurations, as these values grow this work becomes tedious and non-interesting. However it is curious to see how the counts grow and how they distribute among the different symmetry groups. It is clear that as the size of a rosette grows all the counts grow but the counts for the most symmetrical groups grow slower, when compared with the $\mathrm{C} 1$ count.

Table 8 contains the relative frequency of the counts of Table 1 and the asymptotic behavior of these frequencies, as $n$ tends to infinity, showing how the relative growth of the counts behave. Note that we used the expressions $M^{*}(\ldots)$ for these calculations in order to have equiprobability of events. Hence the relative frequencies in the table also give the probability of choosing a particular type of symmetry at random.

From Table 8, one can see that if we were to pick a rosette at random from all possible rosettes, the most likely result would be a $C_{1}$ non-symmetrical rosette. However, as WestphalFitch, Huber, Gómez and Fitch [14] state, humans clearly like to surround themselves with visual patterns that follow some kind of structural order, and if they are asked to select or produce a figure such as a Truchet rosette they will most likely pick a symmetrical one. These authors 


\begin{tabular}{|c|c|c|c|c|c|c|}
\hline $\boldsymbol{G}$ & $\mathbf{1} \times \mathbf{1}$ & $\mathbf{2} \times \mathbf{2}$ & $\mathbf{3} \times \mathbf{3}$ & $\mathbf{4} \times \mathbf{4}$ & $\mathbf{5} \times \mathbf{5}$ & $\mathbf{6} \times \mathbf{6}$ \\
\hline \hline $\boldsymbol{D}_{\mathbf{4}}$ & & $\mathbf{2}$ & & $\mathbf{1 6}$ & & 512 \\
\hline $\boldsymbol{C}_{\mathbf{4}}$ & & $\mathbf{1}$ & & 120 & & 130816 \\
\hline $\boldsymbol{D}_{\mathbf{2}}$ & & $\mathbf{2}$ & & 240 & & 261632 \\
\hline $\boldsymbol{C}_{\mathbf{2}}$ & & $\mathbf{2}$ & & 16200 & & 17179672832 \\
\hline $\boldsymbol{D}_{\mathbf{1}}$ & $\mathbf{1}$ & $\mathbf{1 2}$ & 256 & 65280 & 16777216 & 68719214592 \\
\hline $\boldsymbol{C}_{\mathbf{1}}$ & 0 & $\mathbf{2 4}$ & 32640 & 536830080 & 140737479966720 & 590295810315756109824 \\
\hline Total & $\mathbf{1}$ & $\mathbf{4 3}$ & 32896 & 536911936 & 140737496743936 & 590295810401655390208 \\
\hline
\end{tabular}

Table 5. Counts $N^{*}(G, n, n)$ of square rosettes of square tiles, $n=1, \ldots 6$.

\begin{tabular}{|c|c|c|c|c|c|c|c|c|c|}
\hline $\boldsymbol{G}$ & $\mathbf{1} \times \mathbf{2}$ & $\mathbf{1} \times \mathbf{3}$ & $\mathbf{1} \times \mathbf{4}$ & $\mathbf{1} \times \mathbf{5}$ & $\mathbf{1} \times \mathbf{6}$ & $\mathbf{1} \times \mathbf{7}$ & $\mathbf{1} \times \mathbf{8}$ & $\mathbf{1} \times \mathbf{9}$ & $\mathbf{1} \times \mathbf{1 0}$ \\
\hline \hline $\boldsymbol{C}_{\mathbf{2}}$ & $\mathbf{2}$ & & 8 & & 32 & & 128 & & 512 \\
\hline $\boldsymbol{D}_{\mathbf{1}}$ & $\mathbf{2}$ & & 8 & & 32 & & 128 & & 512 \\
\hline $\boldsymbol{C}_{\mathbf{1}}$ & $\mathbf{2}$ & $\mathbf{1 6}$ & 56 & 256 & 992 & 4096 & 16256 & 65536 & 261632 \\
\hline Total & $\mathbf{6}$ & $\mathbf{1 6}$ & 72 & 256 & 1056 & 4096 & 16512 & 65536 & 262656 \\
\hline
\end{tabular}

Table 6. Counts $N(G, 1, n)$ of rectangular rosettes, $n=1, \ldots 10$.

\begin{tabular}{|c|c|c|c|c|c|c|c|}
\hline $\boldsymbol{G}$ & $\mathbf{2} \times \mathbf{2}$ & $\mathbf{2} \times \mathbf{3}$ & $\mathbf{2} \times \mathbf{4}$ & $\mathbf{2} \times \mathbf{5}$ & $\mathbf{2} \times \mathbf{6}$ & $\mathbf{2} \times \mathbf{7}$ & $\mathbf{2} \times \mathbf{8}$ \\
\hline \hline $\boldsymbol{D}_{\mathbf{2}}$ & $\mathbf{4}$ & & $\mathbf{1 6}$ & & 64 & & 256 \\
\hline $\boldsymbol{C}_{\mathbf{2}}$ & $\mathbf{6}$ & 32 & 120 & 512 & 2016 & 8192 & 32640 \\
\hline $\boldsymbol{D}_{\mathbf{1}}$ & $\mathbf{1 2}$ & 32 & 240 & 512 & 4032 & 8192 & 65280 \\
\hline $\boldsymbol{C}_{\mathbf{1}}$ & 54 & 992 & 16200 & 261632 & 4191264 & 67100672 & 1073692800 \\
\hline Total & 76 & 1056 & 16576 & 262656 & 4197376 & 67117056 & 1073790976 \\
\hline
\end{tabular}

Table 7. Counts $N(G, 2, n)$ of rectangular rosettes, $n=2, \ldots 8$.

\begin{tabular}{|l||c|c|c|c|c|c|}
\hline & $\mathbf{n}=\mathbf{1}$ & $\mathbf{n}=\mathbf{2}$ & $\mathbf{n}=\mathbf{3}$ & $\mathbf{n}=\mathbf{4}$ & $\mathbf{n} \rightarrow \infty, \mathbf{n}$ even & $\mathbf{n} \rightarrow \infty, \mathbf{n}$ odd \\
\hline \hline $\mathbf{D}_{\mathbf{4}}$ & & 0,0078 & & $3,73 \mathrm{E}-09$ & $2^{-\frac{7}{4} n^{2}}$ & \\
\hline $\mathbf{C}_{\mathbf{4}}$ & & 0,0078 & & $5,59 \mathrm{E}-08$ & $2^{-\frac{3}{2} n^{2}}(1+o(1))$ & \\
\hline $\mathbf{D}_{\mathbf{2}}$ & & 0,016 & & $1,12 \mathrm{E}-07$ & $2 \times 2^{-\frac{3}{2} n^{2}}(1+o(1))$ & \\
\hline $\mathbf{C}_{\mathbf{2}}$ & & 0,031 & & $1,51 \mathrm{E}-05$ & $2^{-n^{2}}(1+o(1))$ & \\
\hline $\mathbf{D}_{\mathbf{1}}$ & 1 & 0,19 & 0,0039 & $6,08 \mathrm{E}-05$ & $4 \times 2^{-n^{2}}(1+o(1))$ & $2 \times 2^{-n^{2}}$ \\
\hline $\mathbf{C}_{\mathbf{1}}$ & 0 & 0,75 & 0,996 & 0,99992 & $1-5 \times 2^{-n^{2}}(1+o(1))$ & $1-2 \times 2^{-n^{2}}$ \\
\hline
\end{tabular}

Table 8. Relative frequencies of the counts $M^{*}(G, n, n)$ of square rosettes.

have conducted an experiment where people were asked to spontaneously produce several square rosettes, $6 \times 6$, made up of tiles which are basically an ornamented variant of a Truchet tile. The participants created their rosettes on a touch screen starting from a randomly produced configuration by freely rotating any of the tiles on it. As the authors report, "The majority of patterns produced ( $72 \%$ ) contained at least one type of symmetry, whereas $28 \%$ did not, or only incomplete symmetry. ... The 'non-symmetrical' patterns nonetheless often had a high degree of order so these measures are conservative" [14]. This experiment confirms the general idea that humans have a natural appeal for ordered, symmetrical patterns. In the context of our work we may say that although most Truchet rosettes are non-symmetrical, if we were to choose a rosette of a given size, we would tend to choose one with some kind of symmetry. Nevertheless, random non-symmetrical Truchet rosettes have their own beauty and some of these configurations become quite attractive especially when they form connected paths within themselves. See Figure 8 for some examples. 


\section{Hands-on Truchet rosettes - applied examples}

In this section we present two applications of Truchet rosettes which can be used, for instance, in school activities linking mathematics with art. They are both square shaped paper objects which can be folded/flexed into different configurations. This type of reconfigurable structure, known as a flexagon, usually has the appearance of a hinged polygon. Flexagons can be flexed to display different pairs of faces (back and front), usually in cyclic order, and possibly with more than one cycle within the same flexagon. In this paper we will not go into the mathematical details of flexagons. We suggest [9] and [1] for a deeper reading on the subject. One of the flexagons presented below is known as a square flexagon (or tetraflexagon). We will refer to the other paper object as a square flexagon as well, although not all the configuration obtained are squares.

\subsection{Conventional square flexagon}

The first square flexagon is made out of 24 small squares that after folding are put together in groups of four to form six larger squares. This square flexagon is also known as an hexatetraflexagon because of being composed of six (larger) squares. Figure 33 contains the general net of the square flexagon, front and back sides, showing the six groups of small squares, three on each side.

\begin{tabular}{|c|c|c|}
\hline \begin{tabular}{l|l}
$6 a$ & 0 \\
\end{tabular} & \begin{tabular}{l|l|}
$\mathrm{qZ}$ & $4 \mathrm{a}$
\end{tabular} & $4 \mathrm{~b}$ \\
\hline $6 c$ & & $2 a$ \\
\hline $2 d$ & & $6 \mathrm{~b}$ \\
\hline \begin{tabular}{l|l}
$4 c$ & 4 \\
\end{tabular} & \begin{tabular}{l|l}
$4 \mathrm{~d}$ \\
\end{tabular} & $6 d$ \\
\hline
\end{tabular}

\begin{tabular}{|c|c|c|}
\hline $3 b$ & \begin{tabular}{|l|l|} 
qI & qS \\
\end{tabular} & es \\
\hline $3 d$ & & 1a \\
\hline $1 d$ & & $3 a$ \\
\hline $\mathrm{pS}$ & \begin{tabular}{|l|l|l|} 
IS & I \\
\end{tabular} & $3 c$ \\
\hline
\end{tabular}

Figure 33. Front and back of the square flexagon (general) net.

When assembled the flexagon looks like a square piece of paper (double-sided) but it folds/flexes itself into several different configurations. If each group of four small squares is simply plain colored (using six colors in total) then when flexing we go through six plain colored $2 \times 2$ squares. However, if the squares contain patterns, when flexing we can go through 14 different configurations since four of the groups may appear in two different orientations and the other two may appear in three different orientations. Figure 34 contains the diagram of all the configurations that appear when flexing.

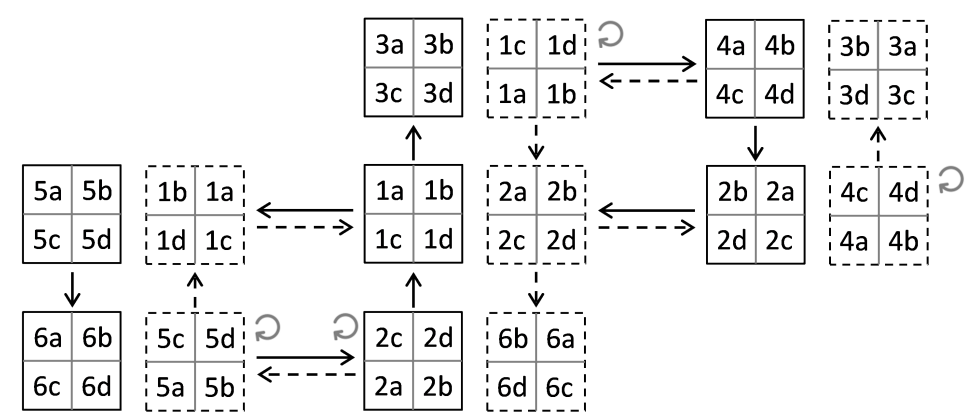

Figure 34. Structure diagram of the square flexagon showing the two cycles. Black contours show the up facing sides and dashed contours the down facing sides; full black arrows indicate the flexing directions (either opening to the sides - horizontal arrow - or opening up and downwards - vertical arrow) starting with the initial front side 1a to 1d; dashed arrows indicate the flexing directions starting with the back side 2 a to $2 \mathrm{~d}$; the "refresh" sign indicates that the view has been rotated a half turn.

To obtain the configurations given in Figure 34 the net of Figure 33 must be folded in a particular direction. Folding through the opposite direction produces a different grouping. The 
folding assumed in Figures 33 and 34 may be done as following: fold the net (upwards) along the bottom line of four squares and turn it $90^{\circ}$ clockwise. Fold again along the same distance from the bottom and turn another $90^{\circ}$ clockwise. Fold and turn again twice. After the last turn you need to flip/squeeze the left hand bottom square into the center of the flexagon so it becomes fixed, with squares $1 \mathrm{a}, 1 \mathrm{~b}, 1 \mathrm{c}$ and $1 \mathrm{~d}$ facing up and resembling a windmill (with $90^{\circ}$ rotational symmetry). The folding of the square flexagon can be seen in https://youtu.be/r3ajakrnHMI.

As can be seen from Figure 34 this flexagon has two cycles. The central configurations using sides numbered 1 and 2 (front and back views) are both bridges between both cycles (also called crossroad points) meaning we can choose two different flexing directions when passing by. All other configurations have only one way in and one way out.

For our square flexagon we used four-by-four Truchet rosettes to fill it. Figure 35 contains the filled net of the square flexagon, front and back images.
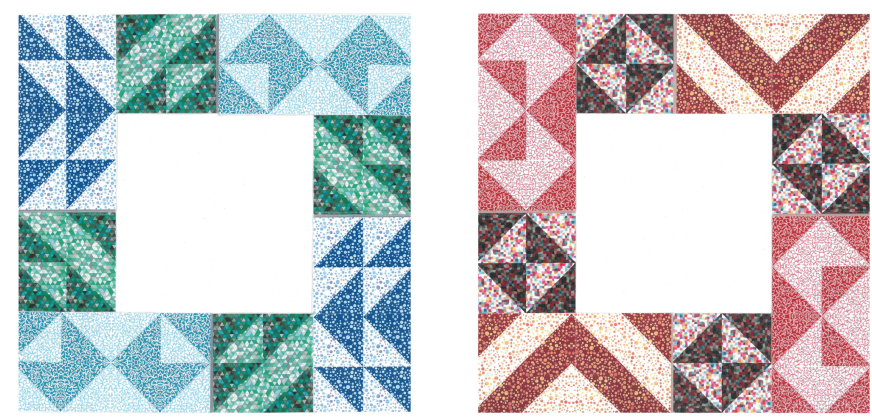

Figure 35. Front and back images of our square flexagon net.

Figure 36 shows the 13 different rosettes that can be viewed when flexing the flexagon after folding as described above (they are not in order of appearance). Although one could obtain 14 different rosettes, we only distinguish 13 because two of them are congruent (this happens because we used very symmetrical rosettes). The following link shows the square flexagon being flexed along its cycles: https://youtu.be/DRPZsxGIgQE.
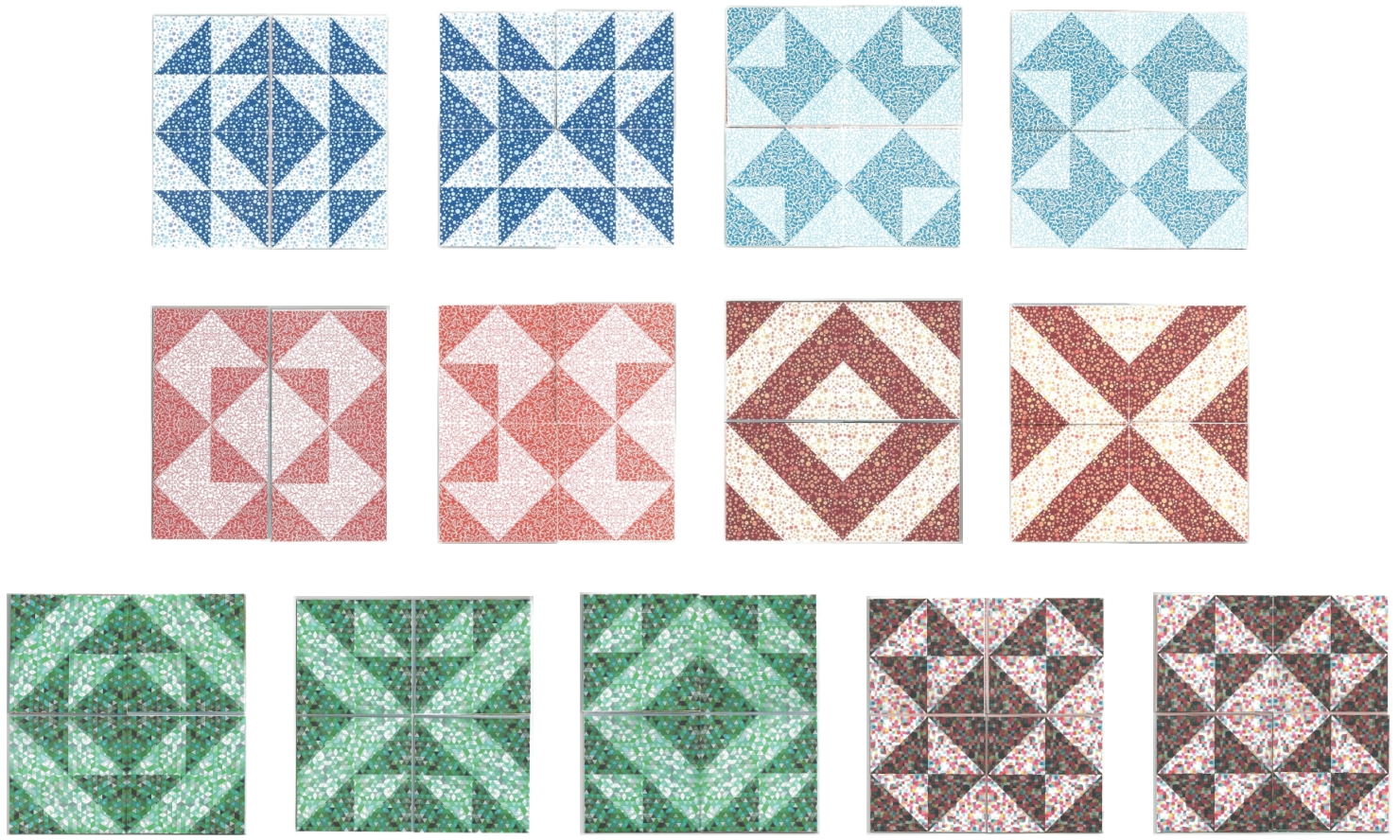

Figure 36. Rosettes emerging from the square flexagon. 


\subsection{Non-conventional square flexagon}

The second square flexagon can be continuously folded until it returns to its original form (one only cycle). The path goes through several squares but also through a cross shape. This paper structure can be found in some children's books written by Consuelo Digón and Cintia Martín [2].

This square flexagon consists of only two square sheets of paper, printed on both sides, which are cut in halves (parallel to one of the sides) and glued together on the corners, as suggested in Figure 37 (the square on the left goes over the right without flipping). The object flexes in a cyclic way through some creases previously done on the sheets, perpendicular to the cuts. The flexagon can be explored on both sides (front and back cycles), giving rise to different figures. Each cycle has four distinct configurations.

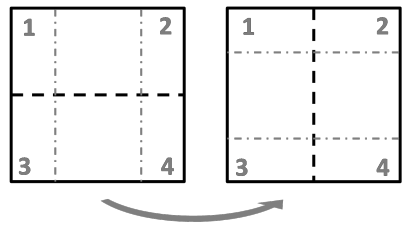

Figure 37. Making a non-conventional square flexagon. Cut lines: black dash lines (note they are perpendicular); creases: grey dash dot lines (a quarter of the side length from the border); grey numbers: gluing points after placing the left-hand square over the right-hand square.

Figure 38 contains the general net of this square flexagon and Figure 39 shows its structure diagram when flexing. We note that in this square flexagon each flexing movement can be done in two steps, the first of which shows half of the starting configuration together with half of the final configuration. In the figures below we do not show these intermediate positions.
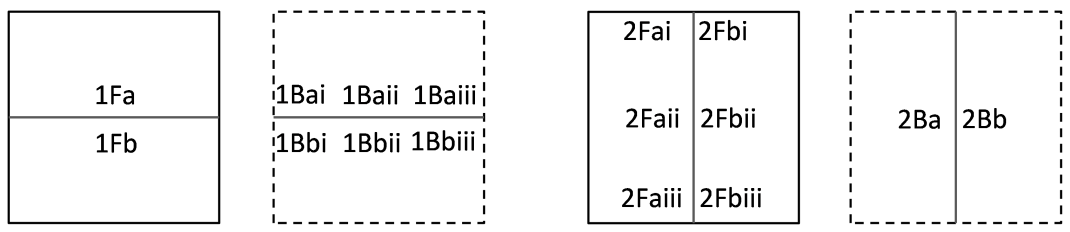

Figure 38. General net of a non-conventional square flexagon. Left to right: left square front and back; right square front and back.
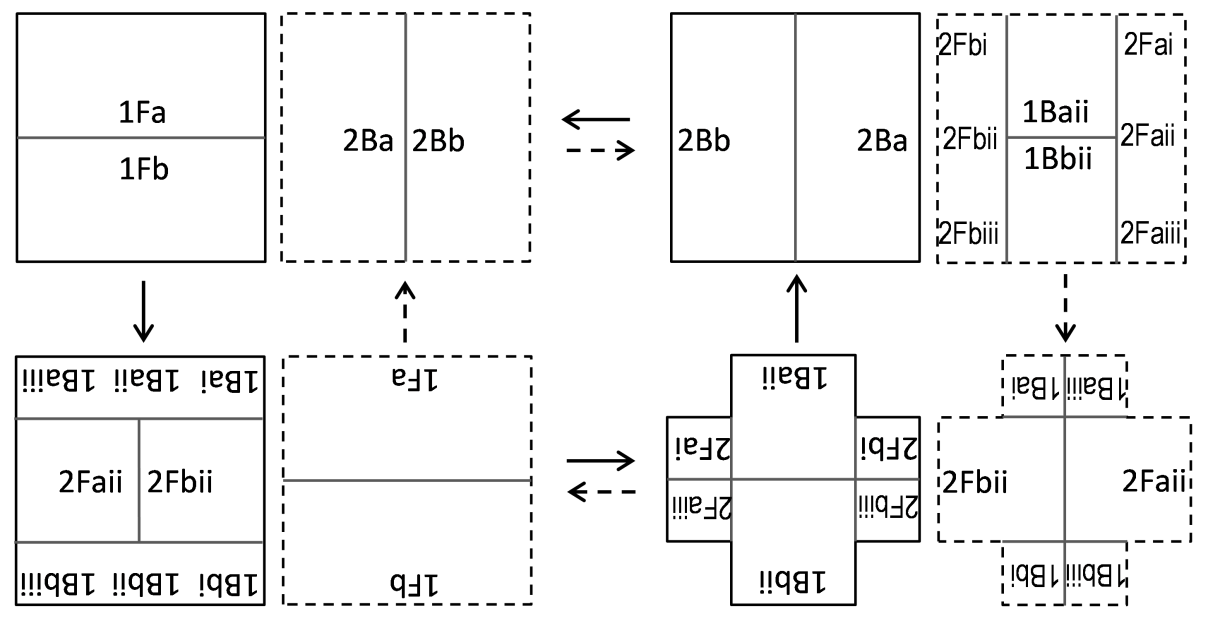

Figure 39. Structure diagram of a non-conventional square flexagon. Black contours show the front cycle sides and dashed contours the back cycle sides; full black arrows indicate the flexing directions (either opening to the sides - horizontal arrow - or opening up and downwards - vertical arrow) strating with the front side $1 \mathrm{Fa}$ and $1 \mathrm{Fb}$; dashed arrows indicate the flexing directions starting with the back side $2 \mathrm{Ba}$ and $2 \mathrm{Bb}$. 
We have constructed two of these square flexagons, using $4 \times 4$ Truchet rosettes. Each of the original squares must be filled on both sides, so four square rosettes are needed for each flexagon, one for each side of each square of paper. The rosettes used are shown in Figure 40. The first set consists of two $D_{4}$ and two $D_{2}$ rosettes. The second set consists of $D_{1}$ rosettes whose reflection axis is parallel to one of the sides of the square.
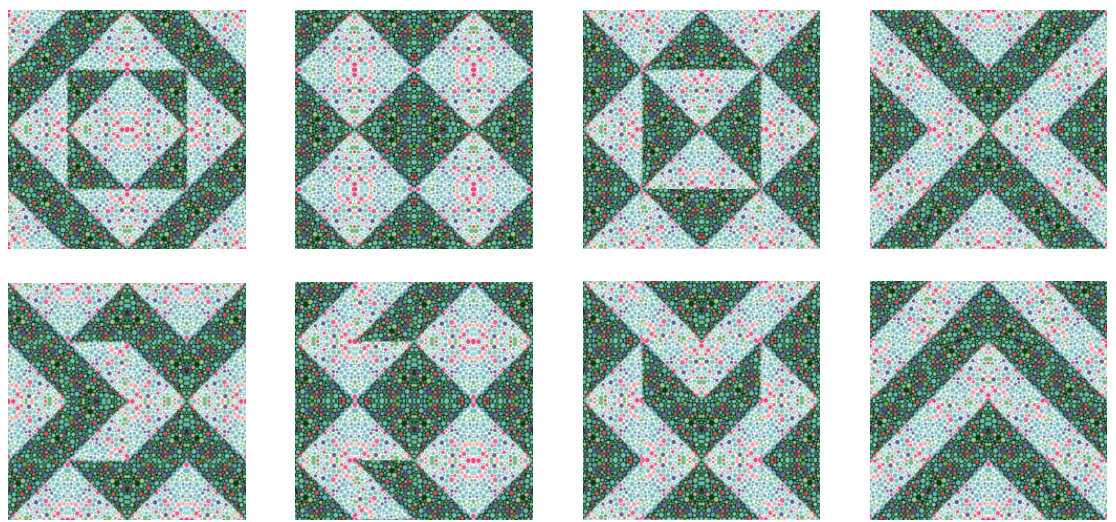

Figure 40. Rosettes composing the non-conventional square flexagons. First row: first square flexagon; second row: second square flexagon. Left to right: left square front and back, right square front and back.

The two sets of four rosettes were glued in groups of two to obtain two pairs of squares printed on both sides. For each pair one of the squares was cut in half horizontally and the other vertically as shown in Figure 41. Creases were made according to Figure 37 and for each pair the left-hand square was glued over the right-hand square.
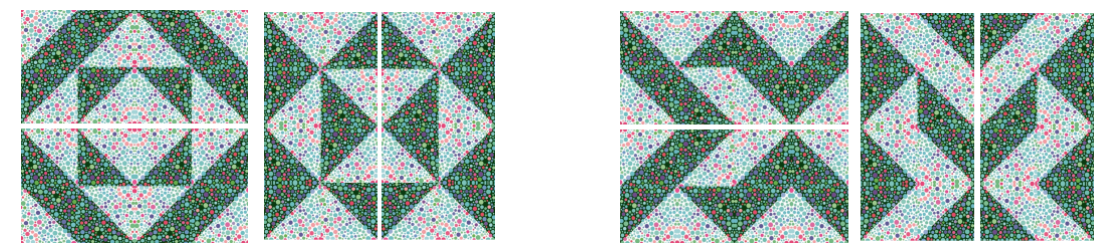

Figure 41. Assembly of the non-conventional square flexagons.

Figures 42 and 43 contain photos of the resulting sequences of rosettes formed as we unfold the square flexagons, in both directions (front and back cycles).
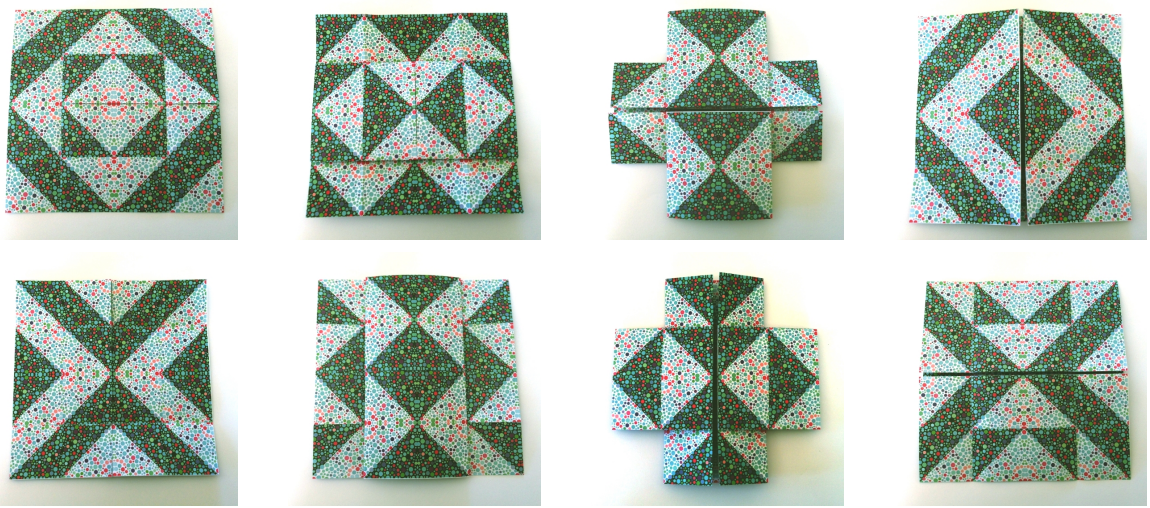

Figure 42. Sequence of rosettes when unfolding the first non-conventional square flexagon. First line — front cycle; second line - back cycle.

The following links show the final result for each flexagon: https://youtu.be/9tOFnsvA5-M and https://youtu.be/hqUS5Gzla6M. 

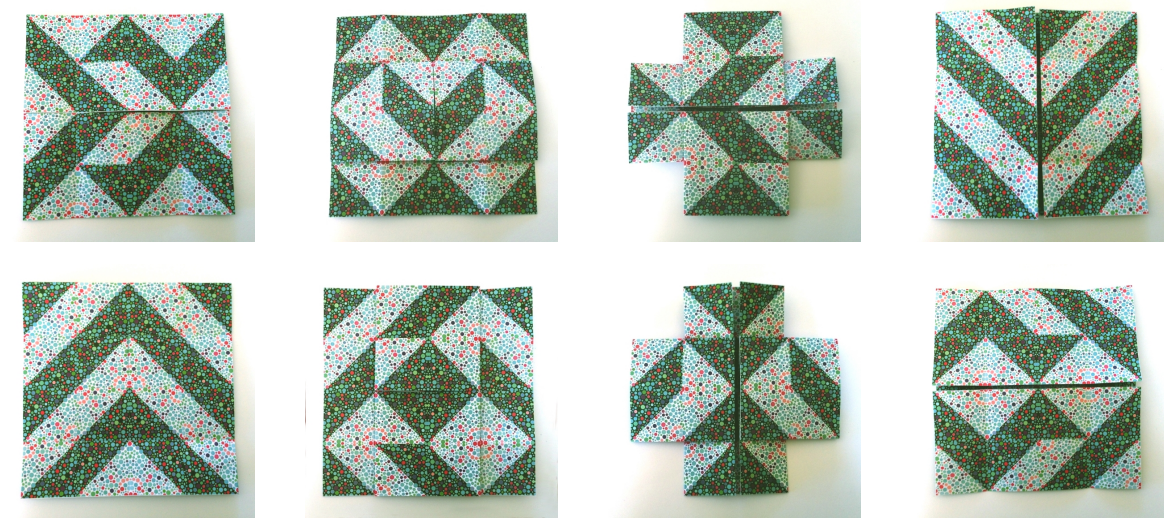

Figure 43. Sequence of rosettes when unfolding the second non-conventional square flexagon. First line — front cycle; second line - back cycle.

These flexagons are easy to create and make a wonderful activity for children to make at school. The resulting configurations while flexing the square flexagon can be very surprising, especially if the starting rosettes are not very symmetrical. Note for instance, that in the second square flexagon described above, the second configuration of the second row of Figure 43 has a mirror symmetry along a diagonal line, although none of the original rosettes had such a mirror symmetry (they all had only one mirror symmetry: either vertical or horizontal).

\subsection{Acknowledgements}

This work was supported by the Portuguese Foundation for Science and Technology (FCTFundação para a Ciência e a Tecnologia), through CIDMA - Center for Research and Development in Mathematics and Applications, within project UID/MAT/04106/2019.

\section{References}

[1] J.Beier and C. Yackel, Groups Associated to Flexagons In J. Rosenhouse and J. Beineke (Eds.), The Mathematics of Various Entertaining Subjects: Research in Recreational Mathematics (pp. 81-94). Princeton: Princeton University Press, 2016.

[2] C. Consuelo Digón and C. Martín, 3 cuentos infinitos: En una cabeza; rase un rey; La manzana, Valladolid: Ediciones Tralarí, 2013.

[3] D. Doüat, Méthode pour faire une infinité de dessins différents, avec des carreaux mi-partis de deux couleurs par une ligne diagonale, Académie Royale des Sciences, Paris, 1722.

[4] A. Hall, Hands-on symmetry, In J. N. Silva (Ed.), Proceedings of the Recreational Mathematics Colloquium IV 2016, Lisbon, Ludus Association, pp. 87-102.

[5] S. Jablan, Symmetry and Ornament, Bridges: Mathematical Connections in Art, Music and Science, Conference Proceedings 2000, pp. 1-13.

[6] S. Jablan, Modularity in Science and Art, VisMath, 4, No.1 (2002). Available at http://www.mi.sanu.ac.rs/vismath/jablan/d3.htm.

[7] E. Lord and S. Ranganathan, Truchet tilings and their generalisations, Resonance 11 (2006), 4250.

[8] G. Martin, Transformation Geometry: An Introduction to Symmetry, New York: Springer-Verlag, 1982.

[9] L.P. Pook, Serious Fun with Flexagons, Springer, 2009.

[10] C. S. Smith and P. Boucher, The Tiling Patterns of Sebastien Truchet and the Topology of Structural Hierarchy, Leonardo 20, No. 4 (1987), pp. 373-385.

[11] P. Stevens, Handbook of Regular Patterns - An Introduction to Symmetry in Two Dimensions, Cambridge: The MIT Press, 1999.

[12] R. Teixeira, Patterns, Mathematics and Culture: The search for symmetry in Azorean sidewalks and traditional crafts, Recreational Mathematics Magazine 3 (2015), pp. 51-72. 
13] D. Washburn and D. Crowe, Symmetries of Culture - Theory and Practice of Plane Pattern Analysis, Seattle: University of Washington Press, 1988.

[14] G. Westphal-Fitch, L. Huber, J. C. Gomez and W. T. Fitch, Production and perception rules underlying visual patterns: effects of symmetry and hierarchy. Phil. Trans. R. Soc. B 367 (2012), pp. 2007-2022. doi:10.1098/rstb.2012.0098 